TRANSACTIONS OF THE

AMERICAN MATHEMATICAL SOCIETY

Volume 354, Number 10, Pages 4153-4178

S 0002-9947(02)02978-1

Article electronically published on June 4, 2002

\title{
AMENABILITY AND EXACTNESS FOR DYNAMICAL SYSTEMS AND THEIR $C^{*}$-ALGEBRAS
}

\author{
CLAIRE ANANTHARAMAN-DELAROCHE
}

\begin{abstract}
We study the relations between amenability (resp. amenability at infinity) of $C^{*}$-dynamical systems and equality or nuclearity (resp. exactness) of the corresponding crossed products.
\end{abstract}

\section{INTRODUCTION}

It has long been known that the amenability of a locally compact group $G$ is strongly connected with the nuclearity of its associated $C^{*}$-algebras. First, it was observed in Guil that the amenability of $G$ implies the nuclearity of its full $C^{*}$ algebra $C^{*}(G)$. The converse is not true since $C^{*}(G)$ is nuclear for every type I or almost connected locally compact group (see $[\mathrm{Co}, \mathrm{Pa} 2$ ). However, Lau and Paterson proved ([LP], $[\mathrm{Pa} 1])$ that if the reduced $C^{*}$-algebra $C_{r}^{*}(G)$ is nuclear and if, in addition, $G$ is inner amenable in the weak sense of [Pa1, §2.35], then $G$ is amenable. Since every discrete group is inner amenable, this gives, in particular, Lance's result [La1] stating that for $G$ discrete, any of its group $C^{*}$-algebras are nuclear if and only if $G$ is amenable.

Similarly, for a locally compact $G$-space $X$, there is a notion of (topological) amenability, which is linked with the nuclearity of the associated crossed products (see [AD1], [ADR, [Re]): if the $G$-space is amenable, then the full crossed product $C^{*}(X \rtimes G)$ is nuclear, and if the reduced crossed product $C_{r}^{*}(X \rtimes G)$ is nuclear with $G$ discrete, then the $G$-space is amenable. A natural notion of inner amenability does not seem to exist in this framework of $G$-spaces. Instead, we introduce a weak notion of inner amenability, that we call property $(\mathrm{W})$, which is expressed in terms of positive type functions with respect to the $G \times G$-space $X \times X$ (Definition 4.3).

We show that a locally compact $G$-space $X$ is amenable if and only if it has property $(\mathrm{W})$ and its reduced crossed product $C^{*}$-algebra $C_{r}^{*}(X \rtimes G)$ is nuclear.

In particular, property $(\mathrm{W})$ makes sense for a locally compact group $G$. It is weaker than inner amenability, but we do not know whether it can be strictly weaker. Every discrete group has property (W) and if $G$ is almost connected (or type I), property (W) is equivalent to its amenability (Remark 5.10). So counterexamples, if they exist, should be sought in the class of totally disconnected groups. An easy observation shows that whenever $G$ has property (W), every $G$-space $X$ has property $(\mathrm{W})$, but the converse is false. More generally, this property can be

Received by the editors March 19, 2001.

2000 Mathematics Subject Classification. Primary 46L05, 46L55.

Key words and phrases. Amenability, Exactness, Nuclearity, $C^{*}$-dynamical systems, Crossed products. 
defined for any locally compact groupoid. It appeared naturally in a joint work in progress with J. Renault concerning exact groupoids.

The above extension to the case of $G$-spaces of the Lau-Paterson result is one of the motivations of this paper. Let us now give a more complete description.

Section 2 sets out a self-contained survey of the notion of amenability for locally compact transformation groups. Although the theory is extensively developed in the monograph $\mathrm{ADR}$ that J. Renault and I have recently written in the more general framework of locally compact groupoids, another motivation for this paper is to give here more direct and easily accessible proofs for readers unfamiliar with groupoids. An advantage is that, when working with transformation groups, one does not need any separability assumption on the locally compact spaces.

This subject has attracted much attention these last three years in connection with the Novikov conjecture for discrete groups, following the works of $\mathrm{Yu}$, HigsonRoe and Higson $([\mathrm{Yu},[\mathrm{HR}],[\mathrm{Hi}])$. In these papers, the crucial notion for a locally compact group is the existence of an amenable action on a compact space. A group with this property will be called amenable at infinity. We briefly study this notion in Section 3 and recall its links with uniform embeddability into Hilbert spaces. In Section 4, we introduce property (W), and Section 5 contains the main results linking the amenability of a $G$-space $X$ to the nuclearity of its associated crossed product $C^{*}$-algebras and to property (W) (Theorems 5.3 and 5.8). Some tentative definitions of amenability for a noncommutative $G$ - $C^{*}$-algebra are proposed in Section 6 , but the situation is still unclear.

In Section 7, we note that our techniques allow us similarly to describe completely the relationships between the exactness of the reduced $C^{*}$-algebra $C_{r}^{*}(G)$ of a locally compact group and its amenability at infinity, in the presence of property $(\mathrm{W})$ : obviously if $G$ is amenable at infinity, then $C_{r}^{*}(G)$ is exact, and the converse is true if, in addition, $G$ has property (W). However, since every almost connected group is amenable at infinity, by considering almost connected nonamenable groups, we see that $G$ can be amenable at infinity without having property $(\mathrm{W})$. We leave open the general problem for any locally compact group: does the exactness of $C_{r}^{*}(G)$ imply the amenability at infinity of $G$ ? A direct proof of the equivalence between the exactitude of $C_{r}^{*}(G)$ and the nuclearity of $C_{r}^{*}(\beta G \rtimes G)$, where one considers the obvious action of $G$, assumed to be discrete, on its Stone-Čech compactification $\beta G$, was obtained by Ozawa $[\mathrm{Oz}$ (see also [GK]).

In Section 8, we show how the ideas of Sinclair and Smith explained in [SS can be adapted to prove directly that for a discrete group action on a locally compact space, the nuclearity of the reduced crossed product $C^{*}$-algebra $C_{r}^{*}(X \rtimes G)$ is equivalent to the amenability of the $G$-space. The advantage is that the proof in this case only uses the notions of completely positive approximations and of positive type functions, and neither the representation theory, nor the characterization of nuclearity in terms of $C^{*}$-tensor products. This observation can be useful in some applications, such as entropy computations. Finally, Section 9 is a survey of some open problems.

This paper is a modified and expanded version of the preprint with the same title which was circulated in April 2000. 


\section{AMENABLE TRANSFORMATION GROUPS}

A transformation group is a left $G$-space $X$, where $X$ is a locally compact space, $G$ is a locally compact group and $(x, s) \mapsto s x$ is a continuous left action from $X \times G$ to $X$. Let us first introduce some notation. We denote by $C_{c}(G)$ the space of complex-valued continuous functions with compact support on $G$, and $\operatorname{Prob}(G)$ will be the set of probability measures on $G$, equipped with the weak*-topology. Given $f \in C_{c}(G), x \in X, m \in \operatorname{Prob}(G)$ and $s \in G$, we set

$$
(s f)(x)=f\left(s^{-1} x\right), \quad(s m)(f)=m\left(s^{-1} f\right) .
$$

If $g \in C_{c}(X \times G)$, then $g^{x}$ will be the map $t \mapsto g^{x}(t)=g(x, t)$, and $g(t)$ will be the map $x \mapsto g(t)(x)=g(x, t)$. Finally, $d t$ denotes the left Haar measure on $G$.

2.1. Definition. We say that the transformation group $(X, G)$ (or the $G$-action on $X$, or the $G$-space $X)$ is amenable if there exists a net $\left(m_{i}\right)_{i \in I}$ of continuous maps $x \mapsto m_{i}^{x}$ from $X$ into the space $\operatorname{Prob}(G)$ such that

$$
\lim _{i}\left\|s m_{i}^{x}-m_{i}^{s x}\right\|_{1}=0
$$

uniformly on compact subsets of $X \times G$.

Such a net $\left(m_{i}\right)_{i \in I}$ will be called an approximate invariant continuous mean (a.i.c.m. for short).

Before giving examples, let us state several equivalent definitions.

2.2. Proposition. The following conditions are equivalent:

(1) $(X, G)$ is an amenable transformation group.

(2) There exists a net $\left(g_{i}\right)_{i \in I}$ of nonnegative continuous functions on $X \times G$ such that

(a) for every $i \in I$ and $x \in X, \int_{G} g_{i}^{x}(t) d t=1$;

(b) $\lim _{i} \int_{G}\left|g_{i}^{s x}(s t)-g_{i}^{x}(t)\right| d t=0$ uniformly on compact subsets of $X \times G$.

(3) There exists a net $\left(g_{i}\right)_{i \in I}$ in $C_{c}(X \times G)^{+}$such that

(a) $\lim _{i} \int_{G} g_{i}^{x} d t=1$ uniformly on compact subsets of $X$;

(b) $\lim _{i} \int_{G}\left|g_{i}^{s x}(s t)-g_{i}^{x}(t)\right| d t=0$ uniformly on compact subsets of $X \times G$.

Proof. $(1) \Rightarrow(2)$. Let $g \in C_{c}(G)^{+}$be such that $\int g(t) d t=1$ and set

$$
g_{i}(x, s)=\int g\left(t^{-1} s\right) d m_{i}^{x}(t) .
$$

By the Fubini theorem, we get $\int g_{i}^{x}(s) d s=1$ for every $x \in X$ and $i \in I$. Moreover, for $(x, s) \in X \times G$, we have

$$
\begin{aligned}
\int\left|g_{i}^{s x}(s t)-g_{i}^{x}(t)\right| d t & =\int\left|\int g\left(u^{-1} s t\right) d m_{i}^{s x}(u)-\int g\left(u^{-1} t\right) d m_{i}^{x}(u)\right| d t \\
& =\int\left|\int g\left(u^{-1} s t\right) d m_{i}^{s x}(u)-\int g\left(u^{-1} s t\right) d\left(s m_{i}^{x}\right)(u)\right| d t \\
& \leq \iint g\left(u^{-1} s t\right) d\left|m_{i}^{s x}-s m_{i}^{x}\right|(u) d t
\end{aligned}
$$

where $\left|m_{i}^{s x}-s m_{i}^{x}\right|$ is the total variation of $m_{i}^{s x}-s m_{i}^{x}$. Using again the Fubini theorem, we obtain the majoration

$$
\leq \int\left[\int g\left(u^{-1} s t\right) d t\right] d\left|m_{i}^{s x}-s m_{i}^{x}\right|(u) \leq\left\|m_{i}^{s x}-s m_{i}^{x}\right\|_{1} .
$$


This tends to 0 uniformly on compact subsets of $X \times G$.

$(2) \Rightarrow(3)$. An easy approximation argument allows us to replace the net $\left(g_{i}\right)_{i \in I}$ by a net in $C_{c}(X \times G)^{+}$satisfying conditions (a) and (b) of (3).

$(3) \Rightarrow(2)$. Let $\left(g_{i}\right)_{i \in I}$ as in (3) and choose $g \in C_{c}(G)^{+}$as above. Let us define $\left(g_{i, n}\right)$ by

$$
g_{i, n}(x, s)=\frac{g_{i}(x, s)+\frac{1}{n} g(s)}{\int g_{i}(x, t) d t+\frac{1}{n}} .
$$

Then $\left(g_{i, n}\right)_{(i, n) \in I \times \mathbb{N}^{*}}$ satisfies conditions (a) and (b) of (2).

$(2) \Rightarrow(1)$ is obvious: given $\left(g_{i}\right)_{i \in I}$ as in (2), we define $m_{i}^{x}$ to be the probability measure with density $g_{i}^{x}$ with respect to the left Haar measure. Then $\left(m_{i}\right)_{i \in I}$ is an a.i.c.m.

2.3. Definition. A complex-valued function $h$ defined on $X \times G$ is said to be of positive type (with respect to the $G$-space $\mathrm{X}$ ) if for every $x \in X$, every positive integer $n$ and every $t_{1}, \ldots, t_{n} \in G, \lambda_{1}, \ldots, \lambda_{n} \in \mathbb{C}$ we have

$$
\sum_{i, j} h\left(t_{i}^{-1} x, t_{i}^{-1} t_{j}\right) \bar{\lambda}_{i} \lambda_{j} \geq 0 .
$$

Similarly, a function $h$ defined on $X \times G$, with values in a $C^{*}$-algebra $A$, is said to be of positive type if for every $x \in X$, every positive integer $n$ and every $t_{1}, \ldots, t_{n} \in G$, the matrix $\left[h\left(t_{i}^{-1} x, t_{i}^{-1} t_{j}\right)\right] \in M_{n}(A)$ is positive.

We denote by $L^{2}(G) \otimes C_{0}(X)$ the completion of $C_{c}(X \times G)$ endowed with the norm

$$
\|\xi\|_{2}=\left(\sup _{x \in X} \int|\xi(x, t)|^{2} d t\right)^{1 / 2} .
$$

Note that $L^{2}(G) \otimes C_{0}(X)$ is a Hilbert $C^{*}$-module over the algebra $C_{0}(X)$ of continuous functions vanishing at infinity, with scalar product

$$
\langle\xi, \eta\rangle(x)=\int \overline{\xi(x, t)} \eta(x, t) d t,
$$

and module structure defined by

$$
(\xi f)(x, s)=\xi(x, s) f(x) .
$$

For the basic facts on Hilbert $C^{*}$-modules we refer the reader to [La2].

To every $\xi \in L^{2}(G) \otimes C_{0}(X)$, we associate the coefficient $(\xi, \xi)$ defined by

$$
(\xi, \xi)(x, s)=\int \overline{\xi(x, t)} \xi\left(s^{-1} x, s^{-1} t\right) d t .
$$

It is a continuous positive type function on $X \times G$, with compact support if $\xi$ is so. The following result is the analogue for transformation groups of a well-known result of Godement [Di, Théorème 13.8.6].

2.4. Lemma. Let $k$ be a continuous function of positive type on $X \times G$, with compact support. There exists $\xi \in L^{2}(G) \otimes C_{0}(X)$ such that $k=(\xi, \xi)$.

Proof. Let $\rho(k)$ be the $C_{0}(X)$-linear bounded endomorphism of $L^{2}(G) \otimes C_{0}(X)$ such that

$$
(\rho(k) \xi)(x, s)=\int k\left(s^{-1} x, s^{-1} t\right) \xi(x, t) d t
$$


for every $\xi \in L^{2}(G) \otimes C_{0}(X)$. Since $k$ is a positive type function, $\rho(k)$ is a positive operator. Let $\left(f_{i}\right)_{i \in I}$ be an approximate unit of $C_{0}(X)$ and $\left(\varphi_{j}\right)_{j \in J}$ be a standard approximate unit of $L^{1}(G)$ (i.e., made of nonnegative continuous functions with compact supports in a basis of neighbourhoods of the unit of $G$ ). We set

$$
\xi_{i, j}=\rho(k)^{1 / 2}\left(f_{i} \otimes \varphi_{j}\right) .
$$

Then $\left(\xi_{i, j}\right)$ is a Cauchy net in $L^{2}(G) \otimes C_{0}(X)$, and if $\xi$ denotes its limit, we have

$$
\begin{aligned}
(\xi, \xi)(x, s) & =\lim _{i, j}\left(\xi_{i, j}, \xi_{i, j}\right)(x, s) \\
& =\lim _{i, j} f_{i}(x) f_{i}\left(s^{-1} x\right) \iint \varphi_{j}(t) \varphi_{j}(r) k\left(t^{-1} x, t^{-1} s r\right) d t d r \\
& =k(x, s) .
\end{aligned}
$$

2.5. Proposition. The following conditions are equivalent:

(1) $(X, G)$ is an amenable transformation group.

(2) There exists a net $\left(\xi_{i}\right)_{i \in I}$ in $C_{c}(X \times G)$ such that

(a) $\lim _{i} \int_{G}\left|\xi_{i}(x, t)\right|^{2} d t=1$ uniformly on compact subsets of $X$;

(b) $\lim _{i} \int_{G}\left|\xi_{i}(s x, s t)-\xi_{i}(x, t)\right|^{2} d t=0$ uniformly on compact subsets of $X \times G$.

(3) There exists a net $\left(h_{i}\right)_{i \in I}$ of positive type functions in $C_{c}(X \times G)$ which tends to 1 uniformly on compact subsets of $X \times G$.

Proof. (1) $\Rightarrow(3)$. Let $\left(g_{i}\right)_{i \in I}$ be a net in $C_{c}(X \times G)^{+}$that fulfills conditions (a) and (b) of (3) in Proposition 2.2. We set $\xi_{i}=\sqrt{g_{i}}$ and $h_{i}=\left(\xi_{i}, \xi_{i}\right)$. Then

$$
h_{i}\left(s^{-1} x, e\right)+h_{i}(x, e)-2 h_{i}(x, s)=\int\left|\xi_{i}\left(s^{-1} x, s^{-1} t\right)-\xi_{i}(x, t)\right|^{2} d t .
$$

The inequality $|a-b|^{2} \leq\left|a^{2}-b^{2}\right|$ for $a, b \geq 0$ gives the majoration

$$
\int\left|\xi_{i}\left(s^{-1} x, s^{-1} t\right)-\xi_{i}(x, t)\right|^{2} d t \leq \int\left|g_{i}\left(s^{-1} x, s^{-1} t\right)-g_{i}(x, t)\right| d t .
$$

Since $h_{i}(x, e)=\int g_{i}(x, t) d t$, we see from $(*)$ and $(* *)$ that $\left(h_{i}\right)_{i \in I}$ goes to 1 uniformly on compact subsets of $X \times G$.

The implication $(3) \Rightarrow(2)$ follows immediately from Lemma 2.4 and $(*)$. We just have to observe that the elements of $L^{2}(G) \otimes C_{0}(X)$ can be approximated by elements of $C_{c}(X \times G)$.

$(2) \Rightarrow(1)$. Let $\left(\xi_{i}\right)_{i \in I}$ as in (2) and set $g_{i}=\left|\xi_{i}\right|^{2}$ for $i \in I$. Using the CauchySchwarz inequality and the inequality

$$
\left.|| a\right|^{2}-|b|^{2}|\leq(|a|+|b|)| a-b \mid,
$$

we get

$$
\begin{aligned}
& \int\left|g_{i}(s x, s t)-g_{i}(x, t)\right| d t \\
& \quad \leq\left[\int\left(\left|\xi_{i}(s x, s t)\right|+\left|\xi_{i}(x, t)\right|\right)^{2} d t\right]^{1 / 2} \times\left[\int\left|\xi_{i}(s x, s t)-\xi_{i}(x, t)\right|^{2} d t\right]^{1 / 2} \\
& \quad \leq\left[\left(\int\left|\xi_{i}(s x, t)\right|^{2} d t\right)^{1 / 2}+\left(\int\left|\xi_{i}(x, t)\right|^{2} d t\right)^{1 / 2}\right] \times\left[\int\left|\xi_{i}(s x, s t)-\xi_{i}(x, t)\right|^{2} d t\right]^{1 / 2} .
\end{aligned}
$$

This last term goes to 0 uniformly on compact subsets of $X \times G$. 
2.6. Remark. Obviously, when $X$ and $G$ are $\sigma$-compact, we may replace nets by sequences in the above statements.

2.7. Examples. (1) If $X$ is reduced to a point, our definition of amenability, under the form given in Proposition 2.2, is the existence of a net $\left(k_{i}\right)_{i \in I}$ of continuous nonnegative functions such that $\int k_{i}(t) d t=1$ for all $i$, and $s \mapsto \int\left|k_{i}(s t)-k_{i}(t)\right| d t$ goes to 0 uniformly on compact subsets of $G$; it is essentially Reiter's condition $\left(P_{1}\right)$ Gre page 44]. The equivalent condition given in Proposition 2.5 (3), namely the existence of a net $\left(h_{i}\right)_{i \in I}$ of continuous positive type compactly supported functions converging to 1 uniformly on compact subsets of $G$, is essentially the weak containment condition introduced by Godement [Gre, page 61].

(2) If $G$ is an amenable group, every transformation group $(X, G)$ is amenable. Indeed, given $\left(k_{i}\right)_{i \in I}$ as in (1) above, we define $m_{i}$ to be the constant map on $X$ whose value is the probability measure with density $k_{i}$ with respect to the left Haar measure. Then $\left(m_{i}\right)_{i \in I}$ is an a.i.c.m.

Note that if $(X, G)$ is amenable and if $X$ has a $G$-invariant probability measure $\mu$, then $G$ is an amenable group. Indeed, let us consider a net $\left(g_{i}\right)_{i \in I}$ as in Proposition $2.2(3)$, and set $k_{i}(t)=\int g_{i}(x, t) d \mu(x)$. Then $\left(k_{i}\right)_{i \in I}$ has the properties stated in (1) above, and therefore $G$ is amenable.

(3) For every locally compact group $G$, its left action on itself is amenable. Indeed, let us choose a nonnegative function $g \in C_{c}(G)^{+}$such that $\int g(t) d t=1$ and set $m^{x}(f)=\int f(t) g\left(x^{-1} t\right) d t$ for every $x \in G$ and every $f \in C_{c}(G)$. Then $x \mapsto m^{x}$ is continuous from $G$ into $\operatorname{Prob}(G)$ and $m^{s x}=s m^{x}$ for every $(x, s) \in G \times G$.

Such an action, having a continuous invariant system of probability measures, is called proper. This is equivalent to the usual properness of the map $(x, t) \mapsto(x, t x)$ from $X \times G$ into $X \times X$ (see ADR Cor. 2.1.17]).

(4) Let $\mathbb{F}_{2}$ be the free group with two generators $a$ and $b$. The boundary $\partial \mathbb{F}_{2}$ is the set of all infinite reduced words $\omega=a_{1} a_{2} \ldots a_{n} \ldots$ in the alphabet $S=\left\{a, a^{-1}, b, b^{-1}\right\}$. There is a natural topology on $\partial \mathbb{F}_{2}$ for which $\partial \mathbb{F}_{2}$ is the Cantor discontinuum, and $\mathbb{F}_{2}$ acts continuously to the left by concatenation. The transformation group $\left(\partial \mathbb{F}_{2}, \mathbb{F}_{2}\right)$ is amenable. Indeed, for $n \geq 1$ and $\omega=a_{1} a_{2} \ldots a_{n} \ldots$, define

$$
\begin{aligned}
m_{n}^{\omega}(\{t\}) & =\frac{1}{n} \quad \text { if } \quad t=a_{1} \ldots a_{k}, \quad k \leq \frac{1}{n} \\
& =0 \quad \text { otherwise. }
\end{aligned}
$$

Then $\left(m_{n}\right)_{n \geq 1}$ is an a.i.c.m.

More generally, every hyperbolic group $\Gamma$ acts amenably on its Gromov boundary $\partial \Gamma$. This was first proved by Adams [Ad], by showing that for every quasi-invariant measure $\mu$ on $\partial \Gamma$, the measured transformation group $(\partial \Gamma, \Gamma, \mu)$ is amenable in the sense of Zimmer. This is equivalent to the (topological) amenability of $(\partial \Gamma, \Gamma)$ defined in these notes (see [ADR, Th. 3.3.7]). More recently, Germain has given a direct proof by constructing explicitly an approximate invariant mean ADR, Appendix B].

(5) Another convenient way to show that transformation groups are amenable is to use the invariance of this notion by Morita equivalence [ADR, Th. 2.2.17]. Let us consider for instance a locally compact group $G$ and two closed subgroups $H$ and $K$. Then the left $H$-action on $G / K$ and the right $K$-action on $H \backslash G$ are Morita equivalent. If $K$ is an amenable group, it follows that $(G / K, H)$ is an amenable transformation group. 


\section{Amenability AT infinity}

3.1. Definition. We say that a locally compact group $G$ is amenable at infinity if it admits an amenable action on a compact space $X$.

3.2. Examples. (1) An amenable locally compact group is amenable at infinity: one can take $X$ reduced to a point.

(2) A discrete group $G$ that is hyperbolic in the sense of Gromov is amenable at infinity since its action on its Gromov boundary is amenable.

(3) Every closed subgroup $H$ of a connected Lie group $G$ is amenable at infinity. Indeed, $G$ possesses a closed amenable subgroup $K$ such that $G / K$ is compact. Then $(G / K, H)$ is an amenable transformation group.

(4) If $G$ is amenable at infinity, so are its closed subgroups.

\subsection{Proposition. Every almost connected group is amenable at infinity.}

Proof. Assume first that $G$ is a connected locally compact group. Then $G$ has a compact normal subgroup $K$ such that $G / K$ is a real Lie group [MZ]. By Example 3.2 (3), the group $G / K$ acts amenably on a compact space $X$. Since the map $G \rightarrow G / K$ is proper, for every positive type function $h \in C_{c}(X \times G / K)$, the function $(x, s) \mapsto h(x, s K)$ has a compact support, and it is of course of positive type. Then, using the characterization of amenable actions in Proposition 2.5 (3), we immediately see that the natural $G$-action on $X$ is amenable.

Assume now that $G$ is almost connected, which means that the quotient group $G / G_{0}$ of $G$ by the connected component of the identity is compact. Let $X$ be a compact amenable $G_{0}$-space. Induction from $G_{0}$ to $G$ provides the compact $G$ space $Y=(G \times X) / G_{0}$. Invariance of amenability by Morita equivalence shows that this $G$-space $Y$ is amenable (see [ADR, Prop. 5.2.5]).

Let us denote by $C_{b}^{u}(G)$ the algebra of bounded left uniformly continuous functions on $G$, that is, such that $\|t f-f\|_{\infty}$ goes to 0 when $t$ goes to the unit element $e$ of $G$. Let $\beta^{u} G$ be the spectrum of this algebra. The left action of $G$ onto $G$ by translation extends uniquely to a left action on $\beta^{u} G$. Note that when $G$ is discrete, $\beta^{u} G$ is the Stone-Čech compactification $\beta G$ of $G$.

3.4. Proposition. Let $G$ be a locally compact group. The following conditions are equivalent:

(1) $G$ is amenable at infinity;

(2) the action of $G$ on $\beta^{u} G$ is amenable.

Proof. Of course we have $(2) \Rightarrow(1)$. Now, assume that there exists an amenable transformation group $(X, G)$ with $X$ compact. Choosing $x_{0} \in X$, the map $s \mapsto s x_{0}$ from $G$ to $X$ extends to a continuous map $p: \beta^{u} G \rightarrow X$, by the universal property of the compactification $\beta^{u} G$. Since $p$ is $G$-equivariant, given an a.i.c.m. $\left(m_{i}\right)_{i \in I}$ for $(X, G)$, the net of maps $y \mapsto m_{i}^{p(y)}$ defines an a.i.c.m. for $\left(\beta^{u} G, G\right)$.

Let us denote by $\theta$ the homeomorphism of $G \times G$ such that $\theta(s, t)=\left(s^{-1}, s^{-1} t\right)$. Let $C_{b, \theta}(G \times G)$ be the algebra of bounded continuous functions $f$ on $G \times G$ such that $f \circ \theta$ is the restriction of a continuous function on $\beta^{u} G \times G$. Note that when $G$ is discrete, $C_{b, \theta}(G \times G)$ is the algebra of bounded functions on $G \times G$.

A set $T$ such that $\left\{s^{-1} t:(s, t) \in T\right\}$ is relatively compact will be called a tube.

An easy reformulation of Propositions 2.2 and 2.5 gives a characterization of amenability at infinity of $G$ which only invokes $G$. Recall that a positive type 
kernel on $G \times G$ is a function $h$ defined on $G \times G$ such that, for every positive integer $n$ and every $t_{1}, \ldots, t_{n} \in G$, the matrix $\left[h\left(t_{i}, t_{j}\right)\right]$ is positive.

3.5. Proposition. Let $G$ be a locally compact group. The following conditions are equivalent:

(1) $G$ is amenable at infinity.

(2) There exists a net $\left(g_{i}\right)$ of nonnegative functions in $C_{b, \theta}(G \times G)$ with support in a tube such that

(a) for each $i$ and each $s, \int_{G} g_{i}(s, t) d t=1$;

(b) $\lim _{i} \int_{G}\left|g_{i}(s, u)-g_{i}(t, u)\right| d u=0$, uniformly on tubes.

(3) There exists a net $\left(\xi_{i}\right)$ of functions in $C_{b, \theta}(G \times G)$ with support in a tube such that

(a) for each $i$ and each $s, \int_{G}\left|\xi_{i}(s, t)\right|^{2} d t=1$;

(b) $\lim _{i} \int_{G}\left|\xi_{i}(s, u)-\xi_{i}(t, u)\right|^{2} d u=0$, uniformly on tubes.

(4) There exists a sequence $\left(h_{i}\right)$ of positive type kernels in $C_{b, \theta}(G \times G)$ with support in a tube such that $\lim _{i} h_{i}=1$, uniformly on tubes.

Proof. now, let To go from Propositions 2.2 and 2.5 to the above statement and conversely, we just need a simple change of variables. Assume for instance the existence of a net $\left(h_{i}\right)$ as in (4) above. For $(s, t) \in G \times G$, we set $k_{i}(s, t)=$ $h_{i}\left(s^{-1}, s^{-1} t\right)$, and we still denote by $k_{i}$ its extension to $\beta^{u} G \times G$. In this way we get a continuous function with compact support on $\beta^{u} G \times G$. For $x \in G$, $t_{1}, \ldots, t_{l} \in G$, we have

$$
k_{i}\left(t_{i}^{-1} x, t_{i}^{-1} t_{j}\right)=h_{i}\left(x^{-1} t_{i}, x^{-1} t_{j}\right) .
$$

It follows that $\left(k_{i}\right)$ is a net of continuous positive type functions on $\beta^{u} G \times G$ with compact support. Moreover, for every compact subset $K \subset G$ we have

$$
\begin{aligned}
\sup _{(x, t) \in \beta^{u} G \times K}\left|k_{i}(x, t)-1\right| & =\sup _{(x, t) \in G \times K}\left|k_{i}(x, t)-1\right| \\
& =\sup _{(x, t) \in G \times K}\left|h_{i}\left(x^{-1}, x^{-1} t\right)-1\right| \\
& =\sup \left\{\left|h_{i}(s, t)-1\right|:(s, t) \in G \times G, s^{-1} t \in K\right\} .
\end{aligned}
$$

Therefore, $\left(h_{i}\right)$ goes to one uniformly on compact subsets of $\beta^{u} G \times G$, and (4) implies (1). The converse is even easier and the other assertions are proved in the same way.

Let us end this section with a proof of the known fact (see [Yu]) that every countable group that is amenable at infinity is uniformly embeddable into a Hilbert space.

3.6. Definition. Let $G$ be a locally compact space. We say that $G$ is uniformly embeddable into a Hilbert space if there exist a Hilbert space $H$ and a map $f: G \rightarrow$ $H$ satisfying the following two conditions:

(a) for every compact subset $K$ of $G$ there exists $R>0$ such that

$$
s^{-1} t \in K \Rightarrow\|f(s)-f(t)\| \leq R ;
$$

(b) for every $R>0$ there exists a compact subset $K$ of $G$ such that

$$
\|f(s)-f(t)\| \leq R \Rightarrow s^{-1} t \in K .
$$


When $G$ is a discrete group with a left-invariant metric $d$ such that $(G, d)$ has bounded geometry, this is the usual notion of uniform embedding into a Hilbert space.

3.7. Proposition. Every $\sigma$-compact locally compact group $G$ that is amenable at infinity is uniformly embeddable into a Hilbert space.

Proof. Let $\left(K_{n}\right)$ be a sequence of compact subsets of $G$ with $\bigcup_{n \geq 1} K_{n}=G$ and $K_{n}$ contained into the interior of $K_{n+1}$ for every $n$.

Consider a sequence $\left(\xi_{n}\right)$ of functions on $G \times G$, with support in tubes such that

(i) $\forall n \geq 1, \forall s \in G, \int_{G}\left|\xi_{n}(s, u)\right|^{2} d u=1$,

(ii) $\forall n \geq 1, \sup \left\{\int_{G}\left|\xi_{n}(s, u)-\xi_{n}(t, u)\right|^{2} d u:(s, t) \in G \times G, s^{-1} t \in K_{n}\right\} \leq \frac{1}{n^{2}}$.

For $s \in G, \xi_{n}^{s}$ will denote the element $t \mapsto \xi_{n}(s, t)$ of $l^{2}(G)$. Let us take $H=$ $L^{2}(G) \otimes l^{2}\left(\mathbb{N}^{*}\right)$ and consider the map

$$
s \mapsto f(s)=\bigoplus_{n \geq 1}\left(\xi_{n}^{s}-\xi_{n}^{e}\right)
$$

from $G$ into $H$. Then we have

$$
\|f(s)-f(t)\|^{2}=\sum_{k}\left\|\xi_{k}^{s}-\xi_{k}^{t}\right\|_{2}^{2}
$$

and if $s^{-1} t \in K_{n}$, we have

$$
\|f(s)-f(t)\|^{2} \leq 4 n+\sum_{k>n} \frac{1}{k^{2}} .
$$

Therefore, condition (a) of Definition 3.6 is satisfied.

Now, let $\psi(n)$ be an integer such that for $1 \leq p \leq n$,

$$
\operatorname{Supp} \xi_{p} \subset\left\{(s, t): s^{-1} t \in K_{\psi(n)}\right\} .
$$

In particular, if $s^{-1} t \notin K_{\psi(n)} K_{\psi(n)}^{-1}$ we have

$$
\operatorname{Supp} \xi_{p}^{s} \cap \operatorname{Supp} \xi_{p}^{t}=\emptyset \quad \text { for } \quad 1 \leq p \leq n,
$$

and thus $\left\|\xi_{p}^{s}-\xi_{p}^{t}\right\|_{2}^{2}=2$. It follows that if $\|f(s)-f(t)\|<\sqrt{2 n}$, we have $s^{-1} t \in$ $K_{\psi(n)} K_{\psi(n)}^{-1}$.

3.8. Remarks. (1) In a recent paper Gro, Gromov has announced the existence of finitely generated discrete groups that are not uniformly embeddable into Hilbert spaces.

(2) Let $G$ be a countable discrete group. It can be shown (see [Yu, GK, Th. 4.1], [STY Prop. 8.6]) that $G$ is uniformly embeddable in a Hilbert space if and only if there exists a sequence $\left(h_{n}\right)$ of bounded positive type kernels on $G \times G$ such that

(a) $\lim _{n} h_{n}=1$ uniformly on tubes;

(b) for every $n$ and every $\varepsilon>0$ there exists a finite subset $K$ of $G$ such that $\left|h_{n}(s, t)\right| \leq \varepsilon$ if $s^{-1} t \notin K$ 
(compare with Proposition 3.5. (4)). It is unknown if amenability at infinity is equivalent to uniform embeddability into Hilbert spaces.

Recall that the Strong Novikov Conjecture with coefficients holds for every finitely generated group that admits a uniform embedding into a Hilbert space ( $\mathrm{Yu}], \underline{\mathrm{Hi}}], \underline{\mathrm{STY}}]$ ).

\section{The property (W)}

4.1. Definition. Let $(X, G)$ be a transformation group. The product group $G \times G$ acts on the product space $X \times X$ by

$$
(s, t) \cdot(x, y)=(s x, t y) .
$$

A positive type function $h$ on $X \times X \times G \times G$ (with respect to the $(G \times G)$-space $X \times X)$ is a positive type function in the sense of Definition 2.3, with respect to the $(G \times G)$-action on $X \times X$. In other words, for every $(x, y) \in X \times X$ and for every $\left(s_{1}, t_{1}\right), \ldots,\left(s_{n}, t_{n}\right) \in G \times G$, the matrix $\left[h\left(s_{i}^{-1} x, t_{i}^{-1} y, s_{i}^{-1} s_{j}, t_{i}^{-1} t_{j}\right)\right]$ is positive.

Let us denote by $X \rtimes G$ the semi-direct product groupoid defined by $(X, G)$ : the range map is the first projection $X \times G \rightarrow X$, the source map is $(x, t) \mapsto t^{-1} x$, and the product is defined by $(x, t)\left(t^{-1} x, s\right)=(x, t s)$. If we view $h$ as a function on $(X \times G) \times(X \times G)$, then it is of positive type as a function defined on the groupoid product of $X \rtimes G$ by itself (see [ADR, page 44]). When $X$ is reduced to a point, we warn the reader that we consider here positive type functions on the group product $G \times G$, and not positive type kernels in the sense of the previous section.

4.2. Definition. We say that a closed subset $A$ of $(X \times G) \times(X \times G)$ is proper with respect to projections or $\pi$-proper if for every compact subset $K$ of $X \times G$, the sets $[K \times(X \times G)] \cap A$ and $[(X \times G) \times K] \cap A$ are compact.

We say that a continuous function $h$ on $(X \times G) \times(X \times G)$ is properly supported if its support is $\pi$-proper.

4.3. Definition. We say that a transformation group $(X, G)$ has property $(\mathrm{W})$ if for every compact subset $K$ of $X \times G$ and for every $\varepsilon>0$ there exists a continuous bounded positive type, properly supported, function $h$ on $(X \times G) \times(X \times G)$ such that $|h(x, t, x, t)-1| \leq \varepsilon$ for all $(x, t) \in K$.

4.4. Examples. (1) Every amenable transformation group $(X, G)$ has property (W). Indeed, given $\varepsilon>0$ and a compact subset $K$ of $X \times G$, let $h$ be a positive type function in $C_{c}(X \times G)$ such that $|h(x, t)-1| \leq \varepsilon$ for all $(x, t) \in K$. Then $(x, s, y, t) \mapsto h(x, s) h(y, t)$ is continuous of positive type and compactly supported on $(X \times G) \times(X \times G)$, and $\left|h(x, t)^{2}-1\right| \leq(2+\varepsilon) \varepsilon$ for $(x, t) \in K$.

(2) Every discrete group has property $(W)$, since the characteristic function of the diagonal $\Delta_{G}$ of $G \times G$ is a properly supported positive type function on the product group $G \times G$.

(3) If $G$ is a locally compact group with the property (W), let us show that every transformation group $(X, G)$ has property $(\mathrm{W})$. Given a compact subset $K_{1} \times K_{2}$ of $X \times G$, and $\varepsilon>0$, let $h$ be a bounded continuous positive type function on the product group $G \times G$, properly supported, such that $|h(t, t)-1| \leq \varepsilon$ for all $t \in K_{2}$. Let $\varphi \in C_{c}(X)$ with values in $[0,1]$, such that $\varphi(x)=1$ for all $x \in K_{1}$. Then $(x, s, y, t) \mapsto g(x, s, y, t)=\varphi(x) \varphi(y) h(s, t)$ is a bounded continuous, properly supported, positive type function such that $|g(x, t, x, t)-1| \leq \varepsilon$ for $(x, t) \in K_{1} \times K_{2}$.

In particular, every discrete transformation group has property $(\mathrm{W})$. 
4.5. Definition. Let us say that a locally compact group $G$ is inner amenable if there exists a mean $m$ on $L^{\infty}(G)$ such that $m\left(t f t^{-1}\right)=m(f)$ for all $f \in L^{\infty}(G)$ and $t \in G$.

This is, for instance, the definition given by Paterson [Pa1, p. 85]. We warn the reader that this is not the usual definition of inner amenability, as introduced by Effros in [Ef]. Our notion of inner amenability was also considered by Kirchberg in Ki , under the name of property $(\mathrm{Z})$.

4.6. Proposition. Every inner amenable locally compact group has property (W).

Proof. Let us denote by $\lambda$ and $\rho$ the left and right regular representations of $G$ respectively. By standard approximation arguments, the existence of a mean that is invariant by inner automorphisms implies the existence of a net $\left(\xi_{i}\right)$ in $C_{c}(G)$ such that $\left\|\xi_{i}\right\|_{2}=1$ and $\left\langle\xi_{i}, \lambda_{t} \rho_{t} \xi_{i}\right\rangle$ goes to one uniformly on compact subsets of $G$. Let us define $h_{i}$ on $G \times G$ by

$$
h_{i}(s, t)=\left\langle\xi_{i}, \lambda_{s} \rho_{t} \xi_{i}\right\rangle
$$

Then $\left(h_{i}\right)$ is a net of properly supported, bounded, continuous, positive type functions, going to one uniformly on compact subsets of the diagonal.

\section{Amenability And CROsSed PRoducts}

Let us recall first a few facts on crossed products. We refer to [Pe, Chap. 7] for more details.

5.1. Definitions. Let $G$ be a locally compact group. A $G-C^{*}$-algebra is a $C^{*}$ algebra $A$ endowed with a continuous action $\alpha$ of $G$. Specifically, $\alpha$ is a homomorphism from $G$ to the group $\operatorname{Aut}(A)$ of automorphisms of $A$, such that for $a \in A$ the map $s \mapsto \alpha_{s}(a)$ is norm continuous. We shall often write $s . a$ for $\alpha_{s}(a)$.

A covariant representation of the $G$ - $C^{*}$-algebra $A$ is a pair $(\pi, \sigma)$ where $\pi$ and $\sigma$ are representations of $A$ and $G$ respectively in a Hilbert space $H$, such that

$$
\sigma(s) \pi(a) \sigma(s)^{-1}=\pi(s . a)
$$

for every $a \in A, s \in G$.

We denote by $C_{c}(G, A)$ the space of continuous functions with compact support from $G$ to $A$, equipped with the product

$$
f * g(s)=\int f(t) \alpha_{t}\left(g\left(t^{-1} s\right)\right) d t
$$

and the involution

$$
f^{*}(s)=\frac{\alpha_{s}\left(f\left(s^{-1}\right)\right)^{*}}{\Delta(s)}
$$

where $\Delta$ is the modular function of $G$.

Given a covariant representation $(\pi, \sigma)$, we set, for $f \in C_{c}(G, A)$,

$$
(\pi \times \sigma)(f)=\int \pi(f(t)) \sigma(t) d t \in \mathcal{B}(H) .
$$

Then $\pi \times \sigma$ is a representation of the involutive algebra $C_{c}(G, A)$. The full crossed product $C^{*}(G, A)$ is the completion of $C_{c}(G, A)$ with respect to the norm

$$
\|f\|=\sup _{(\pi, \sigma)}\|(\pi \times \sigma)(f)\|
$$


where $(\pi, \sigma)$ ranges over all covariant representations of $A$.

Note that $\pi \times \sigma$ extends to a representation of $C^{*}(G, A)$ and that every nondegenerate representation of $C^{*}(G, A)$ arises in this way.

We now view the vector space $C_{c}(G, A)$ as a right $A$-module in the obvious way and define on it the $A$-valued scalar product

$$
\langle f, g\rangle_{A}=\int f(t)^{*} g(t) d t .
$$

As we did previously for $A=C_{0}(X)$, we denote by $L^{2}(G) \otimes A$ (or $L^{2}(G, A)$ ) the Hilbert $C^{*}$-module over $A$ obtained from $C_{c}(G, A)$ by completion. The $C^{*}$-algebra of $A$-linear bounded operators on $L^{2}(G) \otimes A$ admitting an adjoint will be denoted by $\operatorname{End}_{A}\left(L^{2}(G) \otimes A\right)$. We define an involutive homomorphism

$$
\Lambda: C_{c}(G, A) \rightarrow \operatorname{End}_{A}\left(L^{2}(G) \otimes A\right)
$$

by the formula

$$
\Lambda(f) \xi(s)=\int \alpha_{s^{-1}}(f(t)) \xi\left(t^{-1} s\right) d t,
$$

and the reduced crossed product $C_{r}^{*}(G, A)$ is the closure of $\Lambda\left(C_{c}(G, A)\right)$.

Given a nondegenerate representation $\pi: A \rightarrow \mathcal{B}(H)$, the Hilbert space $L^{2}(G) \otimes A \otimes_{A} H$ will be identified with $L^{2}(G, H)$ by the map sending $f \otimes \xi$ to $t \mapsto \pi(f(t)) \xi$. Denote by $\lambda$ the left regular representation of $G$ into $L^{2}(G)$ and by $\tilde{\pi}$ the representation of $A$ into $L^{2}(G, H)$ such that

$$
\tilde{\pi}(a) \xi(t)=\pi\left(\alpha_{t^{-1}}(a)\right) \xi(t), \quad \forall a \in A, \forall t \in G .
$$

Then $\left(\tilde{\pi}, \lambda \otimes 1_{H}\right)$ is a covariant representation of the $G$ - $C^{*}$-algebra $A$. We denote by $\tilde{\pi} \times \lambda\left(\right.$ instead of $\tilde{\pi} \times\left(\lambda \otimes 1_{H}\right)$ ) the corresponding representation of $C^{*}(G, A)$, said to be induced by $\pi$. If $\pi$ is a faithful representation, then $b \mapsto \Lambda(b) \otimes_{A} 1_{H}$ is a faithful representation of $C_{r}^{*}(G, A)$ which coincides with $\tilde{\pi} \times \lambda$ on $C_{c}(G, A)$. It follows that $C_{r}^{*}(G, A)$ is a quotient of $C^{*}(G, A)$ and that every induced representation passes to the quotient.

Let us consider a transformation group $(X, G)$. The $C^{*}$-algebra $C_{0}(X)$ of continuous functions on $X$ vanishing at infinity is a $G$ - $C^{*}$-algebra, with $s . f(x)=f\left(s^{-1} x\right)$ for $s \in G, x \in X, f \in C_{0}(X)$. The corresponding crossed products will be denoted by $C^{*}(X \rtimes G)$ and $C_{r}^{*}(X \rtimes G)$ respectively. When $X$ is reduced to a point, we get the full and reduced $C^{*}$-algebras of $G$, denoted by $C^{*}(G)$ and $C_{r}^{*}(G)$ respectively.

5.2. Definitions. Let $X$ be a locally compact space. A $C_{0}(X)$-algebra is a $C^{*}$ algebra $A$ equipped with a morphism from $C_{0}(X)$ into the center $Z(M(A))$ of the multiplier algebra of $A$, such that $\overline{C_{0}(X) A}=A$.

Let $(X, G)$ be a transformation group. A $G-C_{0}(X)$-algebra is a $G$ - $C^{*}$-algebra $A$ which is also a $C_{0}(X)$-algebra and satisfies the compatibility condition

$$
s .(f a)=(s . f)(s . a), \quad \forall f \in C_{0}(X), a \in A, s \in G .
$$

Given two $C^{*}$-algebras $A$ and $B$, we denote by $A \otimes B$ their minimal $C^{*}$-tensor product, and by $A \otimes_{\max } B$ their maximal $C^{*}$-tensor product.

5.3. Theorem AD1. Let $(X, G)$ be a transformation group, and consider the following conditions:

(1) $(X, G)$ is amenable.

(2) For every $G$ - $C_{0}(X)$-algebra $A, C_{r}^{*}(G, A)=C^{*}(G, A)$. 
(3) For every nuclear $G$ - $C_{0}(X)$-algebra $A, C_{r}^{*}(G, A)$ is nuclear.

(4) $C_{r}^{*}(X \rtimes G)$ is nuclear.

Then $(1) \Rightarrow(2) \Rightarrow(3) \Rightarrow(4)$.

Proof. $(1) \Rightarrow(2)$. Let $(\pi, \sigma)$ be a covariant representation of the $G$ - $C^{*}$-algebra $A$ in $H$. We show that $\pi \times \sigma$ is weakly contained in the representation $\tilde{\pi} \times \lambda$. Let $\left(\xi_{i}\right)$ be a net as in Proposition 2.5 (2). Given $v \in H$, we define, for $t \in G$,

$$
\eta_{i}(t)=\sigma\left(t^{-1}\right) \pi\left(\xi_{i}(t)\right) v .
$$

Here $\xi_{i}(t) \in C_{c}(X)$ is viewed as an element of $Z(M(A))$, and $\pi$ is extended to $M(A)$. For $f \in C_{c}(X \times G)$, a straightforward computation gives

$$
\begin{aligned}
\left\langle\eta_{i},(\tilde{\pi} \times \lambda)(f) \eta_{i}\right\rangle & =\int\left\langle\eta_{i}(s), \pi\left(\alpha_{s^{-1}}(f(t))\right) \eta_{i}\left(t^{-1} s\right)\right\rangle d s d t \\
& =\int\left\langle\pi\left(h_{i}(t)\right) v, \pi(f(t)) \sigma(t) v\right\rangle d t
\end{aligned}
$$

where $h_{i}(x, t)=\int \xi_{i}(x, s) \overline{\xi_{i}\left(t^{-1} x, t^{-1} s\right)} d s$. Since $\left(h_{i}\right)$ goes to 1 uniformly on compact subsets of $X \times G$, we have

$$
\lim _{i}\left\langle\eta_{i},(\tilde{\pi} \times \lambda)(f) \eta_{i}\right\rangle=\langle v,(\pi \times \sigma)(f) v\rangle .
$$

Therefore $\pi \times \sigma$ is weakly contained in $\tilde{\pi} \times \lambda$, and (2) is proved.

$(2) \Rightarrow(3)$. Let $B$ be a $C^{*}$-algebra. Since $A \otimes B$ is a $G-C_{0}(X)$-algebra in an obvious way, we have

$$
C_{r}^{*}(G, A) \otimes B=C_{r}^{*}(G, A \otimes B)=C^{*}(G, A \otimes B)
$$

by (2). Moreover, $A \otimes B=A \otimes_{\max } B$ because $A$ is nuclear. It follows that

$$
\begin{aligned}
C_{r}^{*}(G, A) \otimes B=C^{*}\left(G, A \otimes_{\max } B\right) & =C^{*}(G, A) \otimes_{\max } B \\
& =C_{r}^{*}(G, A) \otimes_{\max } B
\end{aligned}
$$

and therefore $C_{r}^{*}(G, A)$ is nuclear.

$(3) \Rightarrow(4)$ is obvious.

5.4. Lemma. Let $f$ be a bounded, continuous, properly supported, positive type function on $(X \times G) \times(X \times G)$. For $(x, s) \in X \times G$, let us denote by $\tilde{f}_{(x, s)}$ the continuous function with compact support $(y, t) \mapsto(1 / \sqrt{\Delta(t)}) f(x, s, y, t)$ defined on $X \times G$. Then $(x, s) \mapsto \Lambda\left(\tilde{f}_{(x, s)}\right)$ is a positive type function (in the sense of Definition 2.3) with values in $C_{r}^{*}(X \rtimes G)$.

Proof. Let $x \in X$ and $s_{1}, \ldots, s_{n} \in G$ be given. Let us consider elements $\xi_{1}, \ldots, \xi_{n} \in$ $L^{2}(G) \otimes C_{0}(X)$. An easy computation shows that

$$
\begin{aligned}
& \sum_{i, j=1}^{n}\left\langle\xi_{i}, \Lambda\left(\tilde{f}_{\left(s_{i}^{-1} x, s_{i}^{-1} s_{j}\right)}\right) \xi_{j}\right\rangle \\
&=\int \sum_{i, j=1}^{n} \overline{\xi_{i}(y, t)} f\left(s_{i}^{-1} x, s_{i}^{-1} s_{j}, t y, t u^{-1}\right) \xi_{j}(y, u) \frac{d t d u}{\sqrt{\Delta(t u)}} .
\end{aligned}
$$

Then, approximating the measure $\sqrt{\Delta(t)} d t$ by positive measures with finite support, a classical argument shows that the above expression is positive. 
5.5. Definition. Let $(X, G)$ be a transformation group, $B$ a $C^{*}$-algebra and $\Phi$ : $C_{r}^{*}(X \rtimes G) \rightarrow B$ a completely positive map. We say that $\Phi$ has a compact support if there exists a compact subset $K$ of $X \times G$ such that $\Phi(f)=0$ for every $f \in$ $C_{c}(X \times G)$ such that $\operatorname{Supp} f \cap K=\emptyset$.

5.6. Lemma. Let $\Phi: C_{r}^{*}(X \rtimes G) \rightarrow M_{n}(\mathbb{C})$ be a completely positive map. Then for every $\varepsilon>0$ and every finite subset $F$ of $C_{r}^{*}(X \rtimes G)$ there exists a completely positive map $\Psi: C_{r}^{*}(X \rtimes G) \rightarrow M_{n}(\mathbb{C})$, with compact support such that

$$
\|\Psi(a)-\Phi(a)\| \leq \varepsilon \quad \text { for } \quad a \in F .
$$

Proof. Since $\Phi$ is completely positive, by the Stinespring dilatation theorem, there exists a representation $\rho$ of $C_{r}^{*}(X \rtimes G)$ into $H_{\rho}$ and vectors $e_{1}, \ldots, e_{n} \in H_{\rho}$ such that for $a \in C_{r}^{*}(X \rtimes G)$,

$$
\Phi(a)=\left[\left\langle e_{i}, \rho(a) e_{j}\right\rangle\right] \in M_{n}(\mathbb{C}) .
$$

Let us choose a faithful representation $\pi$ of $C_{0}(X)$ in a Hilbert space $H$. Since $\tilde{\pi} \times \lambda$ is a faithful representation of $C_{r}^{*}(X \rtimes G)$ into $L^{2}(G, H)$, given $\eta>0$ there exists a multiple $(\tilde{\pi} \times \lambda) \otimes 1_{K}$ of this representation (that is, $\widetilde{\pi_{K}} \times \lambda_{K}$, where $\pi_{K}=\pi \otimes 1_{K}$ and $\left.\lambda_{K}=\lambda \otimes 1_{H \otimes K}\right)$ and vectors $\xi_{1}, \ldots, \xi_{n}$ in $L^{2}(G, H \otimes K)$ such that

$$
\left|\left\langle e_{i}, \rho(a) e_{j}\right\rangle-\left\langle\xi_{i},\left(\widetilde{\pi_{K}} \times \lambda_{K}\right)(a) \xi_{j}\right\rangle\right|<\eta
$$

for $a \in F$ and $i, j \in\{1, \ldots, n\}$. Moreover, the $\xi_{i}$ 's may be chosen with compact support. Since for $f \in C_{c}(X \times G)$, we have

$$
\left\langle\xi_{i},\left(\widetilde{\pi_{K}} \times \lambda_{K}\right)(f) \xi_{j}\right\rangle=\int\left\langle\xi_{i}(s), \pi_{K}\left(\alpha_{s^{-1}}(f(t))\right) \xi_{j}\left(t^{-1} s\right)\right\rangle d s d t,
$$

we see that the completely positive map

$$
a \mapsto \Psi(a):=\left[\left\langle\xi_{i},\left(\widetilde{\pi_{K}} \times \lambda_{K}\right)(a) \xi_{j}\right\rangle\right]
$$

has a compact support and satisfies $\|\Psi(a)-\Phi(a)\| \leq \varepsilon$ for $a \in F$, provided $\eta$ is small enough.

5.7. Corollary. Let $\Phi$ be a nuclear completely positive type map from $C_{r}^{*}(X \rtimes G)$ into a $C^{*}$-algebra $A$. Then for every $\varepsilon>0$ and every finite subset $F$ of $C_{r}^{*}(X \rtimes G)$ there exists a completely positive map $\Psi: C_{r}^{*}(X \rtimes G) \rightarrow A$, with compact support such that

$$
\|\Psi(a)-\Phi(a)\| \leq \varepsilon \quad \text { for } \quad a \in F .
$$

Proof. This follows immediately from Lemma 5.6 and from the fact that $\Phi=\lim _{i} \Phi_{i}$ in the topology of norm pointwise convergence, each $\Phi_{i}$ admitting a factorization $\Phi_{i}=Q_{i} \circ P_{i}$, where $P_{i}: C_{r}^{*}(X \rtimes G) \rightarrow M_{n_{i}}(\mathbb{C})$ and $Q_{i}: M_{n_{i}}(\mathbb{C}) \rightarrow A$ are completely positive maps.

5.8. Theorem. Let $(X, G)$ be a transformation group. The following conditions are equivalent :

(1) $(X, G)$ is amenable.

(2) $C_{r}^{*}(X \rtimes G)$ is nuclear and $(X, G)$ has property (W). 
Proof. (1) $\Rightarrow(2)$ follows from the previous theorem and from Example 4.4 (1).

$(2) \Rightarrow(1)$. Let $\varepsilon>0$ and a compact subset $K \times F$ of $X \times G$ be given. We want to show the existence of a continuous positive type function $h \in C_{c}(X \times G)$ such that

$$
\forall(x, t) \in K \times F, \quad|h(x, t)-1| \leq \varepsilon .
$$

We first choose a bounded, continuous positive type function $f$ on $(X \times G) \times$ $(X \times G)$, properly supported, such that

$$
\forall(x, s) \in K \times F, \quad|f(x, s, x, s)-1| \leq \varepsilon / 3 .
$$

Given $\xi \in L^{2}(G) \otimes C_{0}(X)$ and $s \in G$, let us denote by $R_{s} \xi$ the element $(x, t) \mapsto$ $\xi\left(s^{-1} x, t s\right) \sqrt{\Delta(s)}$. Note that $R_{s}$ does not belong to $\operatorname{End}_{C_{0}(X)}\left(L^{2}(G) \otimes C_{0}(X)\right)$ and that

$$
\left\langle R_{s} \xi, \eta\right\rangle(x)=\left\langle\xi, R_{s^{-1}} \eta\right\rangle\left(s^{-1} x\right)
$$

for $\xi, \eta \in L^{2}(G) \otimes C_{0}(X)$.

Let $\Phi: C_{r}^{*}(X \rtimes G) \rightarrow C_{r}^{*}(X \rtimes G)$ be a completely positive contraction with compact support and let $\xi \in L^{2}(G) \otimes C_{0}(X)$. We define $h$ on $X \times G$ by

$$
h(x, s)=\left\langle R_{s^{-1}} \xi, \Phi\left(\tilde{f}_{(x, s)}\right) \xi\right\rangle\left(s^{-1} x\right) .
$$

Let us observe that $h$ is a continuous positive type function with compact support. First, since $(x, s) \mapsto \tilde{f}_{(x, s)}$ is a continuous function from $X \times G$ into $C_{r}^{*}(X \rtimes G)$, we see that $h$ is continuous. Moreover, using the facts that $f$ is properly supported and that $\Phi$ has a compact support, we get that $h$ is compactly supported. Finally, a straightforward computation gives

$$
h\left(s^{-1} x, s^{-1} t\right)=\left\langle R_{s} \xi, \Phi\left(\tilde{f}_{\left(s^{-1} x, s^{-1} t\right)}\right) R_{t} \xi\right\rangle(x),
$$

the crucial point being that $\Phi\left(\tilde{f}_{\left(s^{-1} x, s^{-1} t\right)}\right) \in C_{r}^{*}(X \rtimes G)$ commutes with $R_{t}$. Since $(x, t) \mapsto \Phi\left(\tilde{f}_{(x, t)}\right)$ is completely positive, we see that $h$ is of positive type.

Let us explain now how we choose $\xi$ and $\Phi$. Let us take a compact neighbourhood $V$ of $e$ in $G$, small enough such that

$$
\left|f\left(x, s, v x, v s v_{1}\right)-f(x, s, x, s)\right| \leq \varepsilon / 3, \quad \forall\left(x, s, v, v_{1}\right) \in K \times F \times V \times V^{-1} .
$$

We consider a nonnegative continuous function $\psi$ with support in $V$ such that $\int \frac{\psi\left(t^{-1}\right)}{\sqrt{\Delta(t)}} d t=1$. Let $\varphi: X \rightarrow[0,1]$ be a continuous function with compact support such that $\varphi(x)=1$ for all $x \in K \cup F^{-1} K$. Then $\xi$ will be the element $(x, t) \mapsto \varphi(x) \psi(t)$ of $L^{2}(G) \otimes C_{0}(X)$.

Since the set $\left\{\tilde{f}_{(x, s)}:(x, s) \in K \times F\right\}$ is compact in $C_{r}^{*}(X \rtimes G)$, given $\eta>0$, we can choose $\Phi$, thanks to Corollary 5.7, such that

$$
\left.\| \Phi\left(\tilde{f}_{(x, s)}\right)-\tilde{f}_{(x, s)}\right) \|_{r} \leq \eta \quad \forall(x, s) \in K \times F,
$$

where $\|\cdot\|_{r}$ denotes the norm in the reduced crossed product. Let us take $\eta$ such that $\eta \sup _{x \in X}\left(\int|\xi(x, u)|^{2} d u\right) \leq \varepsilon / 3$. It follows that

$$
\left|h(x, s)-\left\langle R_{s^{-1}} \xi, \Lambda\left(\tilde{f}_{(x, s)}\right) \xi\right\rangle\left(s^{-1} x\right)\right| \leq \varepsilon / 3 \quad \forall(x, s) \in K \times F .
$$


Observe now that

$$
\left\langle R_{s^{-1}} \xi, \Lambda\left(\tilde{f}_{(x, s)}\right) \xi\right\rangle\left(s^{-1} x\right)=\int \frac{\xi\left(x, u s^{-1}\right)}{\sqrt{\Delta(s)}} \frac{\xi\left(s^{-1} x, w^{-1} u\right)}{\sqrt{\Delta(w)}} f\left(x, s, u s^{-1} x, w\right) d u d w
$$

and that for $(x, s) \in K \times F$,

$$
\begin{aligned}
\int \frac{\xi\left(x, u s^{-1}\right)}{\sqrt{\Delta(s)}} \frac{\xi\left(s^{-1} x, w^{-1} u\right)}{\sqrt{\Delta(w)}} d u d w & =\int \frac{\psi\left(u s^{-1}\right)}{\sqrt{\Delta(s)}} \frac{\psi\left(w^{-1} u\right)}{\sqrt{\Delta(w)}} d u d w \\
& =\int \frac{\psi\left(u s^{-1}\right)}{\sqrt{\Delta(s)}}\left[\int \frac{\psi\left(w^{-1}\right)}{\sqrt{\Delta(w)} \sqrt{\Delta(u)}} d w\right] d u \\
& =\left(\int \frac{\psi\left(t^{-1}\right)}{\sqrt{\Delta(t)}} d t\right)^{2}=1
\end{aligned}
$$

Hence we have, for $(x, s) \in K \times F$,

$$
\begin{aligned}
\mid h(x, s) & -f(x, s, x, s) \mid \\
& \leq \int\left|f\left(x, s, u s^{-1} x, w\right)-f(x, s, x, s)\right| \frac{\psi\left(u s^{-1}\right)}{\sqrt{\Delta(s)}} \frac{\psi\left(w^{-1} u\right)}{\sqrt{\Delta(w)}} d u d w+\varepsilon / 3 .
\end{aligned}
$$

But inside the integral, we have $u s^{-1} \in V$ and $w^{-1} u \in V$, and writing $w=$ $u s^{-1} s u^{-1} w$, we get, by the choice of $V$, that $|h(x, s)-f(x, s, x, s)| \leq 2 \varepsilon / 3$ for all $(x, s) \in K \times F$.

Finally, it follows that $|h(x, s)-1| \leq \varepsilon$ for all $(x, s) \in K \times F$.

As a consequence of the previous theorem, a group $G$ is amenable if and only if its reduced $C^{*}$-algebra is nuclear and $G$ has property $(\mathrm{W})$. In fact, in this case a stronger result is obtained in the next proposition.

5.9. Proposition. A locally compact group $G$ is amenable if and only if it has property $(\mathrm{W})$ and its von Neumann algebra $\mathcal{L}(G)$ is injective.

Proof. Recall that $\mathcal{L}(G)$ is the von Neumann algebra generated in $\mathcal{B}\left(L^{2}(G)\right.$ ) by the left translation operators $\lambda_{s}, s \in G$. Assume that $\mathcal{L}(G)$ is injective and that $G$ has property $(\mathrm{W})$. The proof of Theorem 5.8 can be easily adapted. Given $\xi \in L^{2}(G)$, a bounded continuous positive type function $f$ on the group $G \times$ $G$, properly supported, and a completely positive map $\Phi: C_{r}^{*}(G) \rightarrow \mathcal{L}(G)$, with compact support, we define $h: G \rightarrow \mathbb{C}$ by

$$
h(s)=\left\langle\rho_{s^{-1}} \xi, \Phi\left(\tilde{f}_{s}\right) \xi\right\rangle
$$

where $\left(\rho_{s} \xi\right)(t)=\xi(t s) \sqrt{\Delta(s)}$ and $\tilde{f}_{s}(t)=(1 / \sqrt{\Delta(t)}) f(s, t)$. Then $h$ is a continuous positive type function on $G$, with compact support.

Let $\varepsilon>0$ and a compact subset $F$ of $G$ be given. We choose $\xi$ and $f$ as in the proof of the previous theorem, $X$ being here reduced to a point. Note that $\left\{\rho_{s^{-1}} \xi: s \in F\right\}$ and $\left\{\tilde{f}_{s}: s \in F\right\}$ are compact in the normed spaces $L^{2}(G)$ and $C_{r}^{*}(G) \subset \mathcal{L}(G)$ respectively. Since $\mathcal{L}(G)$ is injective, there is a net $\left(\Phi_{i}\right)$ of completely positive contractions from $C_{r}^{*}(G)$ to $\mathcal{L}(G)$ which factorize through matrix algebras and converge to the inclusion $C_{r}^{*}(G) \rightarrow \mathcal{L}(G)$ in the topology of pointwise 
weak convergence. Using Lemma 5.6, we can find $\Phi: C_{r}^{*}(G) \rightarrow \mathcal{L}(G)$ compactly supported such that

$$
\left|\left\langle\rho_{s^{-1}} \xi, \Phi\left(\tilde{f}_{s}\right) \xi\right\rangle-\left\langle\rho_{s^{-1}} \xi, \Lambda\left(\tilde{f}_{s}\right) \xi\right\rangle\right| \leq \varepsilon / 3
$$

for every $s \in F$. Then we conclude as in the proof of Theorem 5.8.

5.10. Remark. Let us remark that Lau and Paterson $[\mathrm{LP}$ obtained the following similar result: $G$ is amenable if and only if $\mathcal{L}(G)$ is an injective von Neumann algebra and $G$ is inner amenable. More generally, given a quasi-invariant measure $\mu$ on a locally compact $G$-space $X$, they showed that the following conditions are equivalent: (a) $G$ is amenable; (b) the von Neumann crossed product $L^{\infty}(X, \mu) \rtimes G$ is injective, $L^{\infty}(X, \mu)$ has a $G$-invariant mean, and $G$ is inner amenable.

Observe that for every almost connected or type I locally compact group $G$, the full $C^{*}$-algebra $C^{*}(G)$ is nuclear (see [Pa2]). The case of an almost connected group relies on Connes' result stating the nuclearity of $C^{*}(G)$ for every separable connected locally compact group. By Theorem 5.8, these groups do not have property $(\mathrm{W})$ unless they are amenable.

\section{Amenable $G$-algebras}

At present it is not clear what should be the right definition of amenability for a $G$ - $C^{*}$-algebra $A$. Certainly it should imply that $C_{r}^{*}(G, A)=C^{*}(G, A)$, and imply also that $C_{r}^{*}(G, A)$ is nuclear under the assumption that $A$ is nuclear.

Let us observe that the equality of both crossed products is not a functorial property. More precisely, given an equivariant morphism $\Phi$ from a $G$ - $C^{*}$-algebra $A$ into a $G$ - $C^{*}$-algebra $B$, it may happen that $C_{r}^{*}(G, A)=C^{*}(G, A)$ and $C_{r}^{*}(G, B) \neq$ $C^{*}(G, B)$. Let us consider, for example, a discrete group $G, A=C_{0}(G)$ and $B=\mathcal{K}\left(\ell^{2}(G)\right)$, respectively the $C^{*}$-algebra of functions on $G$ vanishing at infinity and the $C^{*}$-algebra of compact operators. We let $G$ act on $B$ by $t \mapsto \operatorname{Ad} \lambda_{t}$, and on $A$ by restriction. This latter action is induced by the proper action of $G$ onto itself by translation, and therefore $C_{r}^{*}(G, A)=C^{*}(G, A)$. On the other hand, one has $C_{r}^{*}(G, B)=B \otimes C_{r}^{*}(G)$ and $C^{*}(G, B)=B \otimes C^{*}(G)$, and therefore these two $C^{*}$-algebras coincide only if $G$ is amenable.

It seems reasonable to include in the definition of amenability for $G$ - $C^{*}$-algebras the following functoriality assumption. We do not know whether it is redundant.

6.1. Definition. We say that a $G$-action on a $C^{*}$-algebra $A$ is weakly amenable (or that the $G$ - $C^{*}$-algebra $A$ is weakly amenable) if for every $G$ - $C^{*}$-algebra $B$, we have $C^{*}(G, A \otimes B)=C_{r}^{*}(G, A \otimes B)$ with respect to the diagonal action.

We say that the $G$-action is strongly amenable if there exists an amenable $G$ space $X$ such that $A$ is a $G-C_{0}(X)$-algebra.

We do not have any reason to choose the spatial tensor product, instead of the maximal one, in the definition of weak amenability. A more natural definition could be to require the functoriality property stated in 9.2 (c) below.

Clearly, Theorem 5.3 implies that every strongly amenable $G$ - $C^{*}$-algebra is weakly amenable.

6.2. Definition. Let $\alpha: G \rightarrow \operatorname{Aut}(A)$ be a morphism from a group $G$ into the automorphism group of a $C^{*}$-algebra $A$. We say that a function $h: G \rightarrow A$ is of positive type with respect to $\alpha$ if for every positive integer $n$ and every $t_{1}, \ldots, t_{n} \in G$ the matrix $\left[\alpha_{t_{i}}\left(h\left(t_{i}^{-1} t_{j}\right)\right)\right] \in M_{n}(A)$ is positive. 
6.3. Proposition. A transformation group $(X, G)$ is amenable if and only if the $G$-C $C^{*}$-algebra $C_{0}(X)$ is strongly amenable.

Proof. Assume that the $G-C^{*}$-algebra $C_{0}(X)$ is strongly amenable. There exist an amenable $G$-space $Y$ and a $G$-equivariant morphism from $C_{0}(Y)$ into $C_{b}(X)$ (the algebra of bounded continuous functions on $X)$ such that $\overline{C_{0}(Y) C_{0}(X)}=C_{0}(X)$. Let $\left(h_{i}\right)_{i \in I}$ be a net of positive type functions in $C_{c}(Y \times G)$ that goes to 1 uniformly on compact subsets of $Y \times G$.

Let us view every element $h$ of $C_{c}(Y \times G)$ as a continuous function from $G$ into $C_{0}(Y)$. Then $h$ is of positive type in the sense of Definition 2.3 if and only if for every positive integer $n$ and every $t_{1}, \ldots, t_{n} \in G$ the matrix $\left[\alpha_{t_{i}}\left(h\left(t_{i}^{-1} t_{j}\right)\right)\right]$ is positive in $M_{n}\left(C_{0}(Y)\right)$, where $\alpha$ is the induced action of $G$ on $C_{0}(Y)$. Let $\varphi$ be a continuous nonnegative function on $X$ with compact support. Then $t \mapsto h(t) \varphi$ defines an element of $C_{c}(G \times X)$, which is of positive type if $h$ is. Indeed, the matrix $\left[\alpha_{t_{i}}\left(h\left(t_{i}^{-1} t_{j}\right)\right) \alpha_{t_{i}}(\varphi)\right]$ is then positive since a coefficientwise product of two positive matrices with coefficients in a commutative $C^{*}$-algebra is still positive. Let us consider an approximate unit $\left(\varphi_{\lambda}\right)_{\lambda \in \Lambda}$ in $C_{0}(X)$ consisting of nonnegative functions with compact support. Then $\left(h_{i} \varphi_{\lambda}\right)_{(i, \lambda) \in I \times \Lambda}$ is a net of positive type functions in $C_{c}(X \times G)$ going to one uniformly on compact subsets. Therefore $(X, G)$ is an amenable transformation group.

The converse is obvious.

In connection with the comparison problem of weak and strong amenability for $G$ - $C^{*}$-algebras, let us recall the following result, which is essentially contained in AD1.

6.4. Proposition. Let $A$ be a $G$-C $C^{*}$-algebra with $A$ nuclear and $G$ discrete. The following conditions are equivalent:

(1) The $G$-action on $A$ is weakly amenable.

(2) The $C^{*}$-algebra $C_{r}^{*}(G, A)$ is nuclear.

(3) There exists a net $\left(h_{i}\right)$ of positive type functions from $G$ to $Z\left(A^{\prime \prime}\right)$ (the centre of the envelopping von Neumann algebra of $A$, viewed as a $G$-C $C^{*}$-algebra) with finite support such that

(a) $h_{i}(e)=1$ for all $i$;

(b) for all $s \in G, \lim _{i} h_{i}(s)=1$ in the $\sigma$-weak topology of $A^{\prime \prime}$.

Proof. For the proof of $(1) \Rightarrow(2)$ see Theorem 5.3, and for the proof of $(2) \Rightarrow$ (3) we refer to [AD1 Théorème 4.5]. Let us prove that $(3) \Rightarrow(1)$. Using Lemma 2.4 , we may write $h_{i}(s)=\left\langle\xi_{i}, \tilde{\alpha}_{s} \xi_{i}\right\rangle$ where $\xi_{i} \in \ell^{2}(G) \otimes Z\left(A^{\prime \prime}\right)$ and $\tilde{\alpha}_{s} \xi_{i}(t)=$ $\alpha_{s}\left(\xi_{i}\left(s^{-1} t\right)\right)$. Here $\alpha$ denotes the $G$-action on $A$ as well as its extension to $A^{\prime \prime}$. Following the same pattern as in the proof of $(1) \Rightarrow(2)$ in Theorem 5.3 , given a covariant representation $(\pi, \sigma)$ of the $G$ - $C^{*}$-algebra $A$ into $H$, and $v \in H$, we define $\eta_{i}(t)=\sigma\left(t^{-1}\right) \pi\left(\xi_{i}(t)\right) v$, where $\pi$ is extended to $A^{\prime \prime}$. In the same way, we conclude that for every $f \in C^{*}(G, A)$ we have

$$
\lim _{i}\left\langle\eta_{i},(\tilde{\pi} \times \lambda)(f) \eta_{i}\right\rangle=\langle v,(\pi \times \lambda)(f) v\rangle .
$$

It follows that $C_{r}^{*}(G, A)=C^{*}(G, A)$.

Finally, let $B$ be another $G$ - $C^{*}$-algebra. Then net $\left(h_{i} \otimes 1\right)$ has the same properties (a), (b) with respect to the $G$ - $C^{*}$-algebra $A \otimes B$, and therefore $C_{r}^{*}(G, A \otimes B)=$ $C^{*}(G, A \otimes B)$. 
6.5. Remark. In order to get the strong amenability in the previous proposition, we need to show that the $h_{i}$ 's can be taken with values in $Z(M(A))$ instead of $Z\left(A^{\prime \prime}\right)$ and replace the $\sigma$-weak topology by the strict topology.

\section{Amenability at Infinity And EXACTNESS}

Although there is an obvious notion of amenability at infinity for transformation groups, we do not develop that theory, since we do not know at the moment any example not having this property. This notion, in the more general framework of locally compact groupoids, where counterexamples exist, will be the subject of a forthcoming paper. In this section we shall only consider the case of locally compact groups.

7.1. Definition $[\mathrm{KW}]$. We say that a locally compact group $G$ is exact if for every $G$-equivariant exact sequence

$$
0 \longrightarrow I \longrightarrow A \longrightarrow A / I \longrightarrow 0
$$

of $G$ - $C^{*}$-algebras, the sequence

$$
0 \longrightarrow C_{r}^{*}(G, I) \longrightarrow C_{r}^{*}(G, A) \longrightarrow C_{r}^{*}(G, A / I) \longrightarrow 0
$$

is exact.

Recall also that the corresponding sequence with the full crossed products instead of the reduced ones is automatically exact. For the basic facts on exact $C^{*}$-algebras, we refer to $\mathrm{Wa}$.

7.2. Theorem. Let $G$ be a locally compact group and consider the following conditions :

(1) $G$ is amenable at infinity.

(2) $G$ is exact.

(3) For every exact $G$ - $C^{*}$-algebra $B$, the crossed product $C_{r}^{*}(G, B)$ is an exact $C^{*}$-algebra.

(4) $C_{r}^{*}(G)$ is an exact $C^{*}$-algebra.

Then $(1) \Rightarrow(2) \Rightarrow(3) \Rightarrow(4)$.

Proof. (1) $\Rightarrow(2)$. Assume that $(Y, G)$ is an amenable transformation group with $Y$ compact. Let $0 \rightarrow I \rightarrow A \rightarrow A / I \rightarrow 0$ be a $G$-equivariant exact sequence of $G-C^{*}$-algebras. Then

$$
0 \rightarrow C(Y) \otimes I \rightarrow C(Y) \otimes A \rightarrow C(Y) \otimes A / I \rightarrow 0
$$

is obviously a $G$-equivariant exact sequence of $G$ - $C(Y)$-algebras when these algebras are endowed with the diagonal $G$-actions. The corresponding sequence

$$
0 \rightarrow C^{*}(G, C(Y) \otimes I) \rightarrow C^{*}(G, C(Y) \otimes A) \rightarrow C^{*}(G, C(Y) \otimes A / I) \rightarrow 0
$$

of full crossed products is exact. On the other hand, by Theorem 5.3, the full and reduced crossed products are the same. Then we obtain the following commutative diagram:

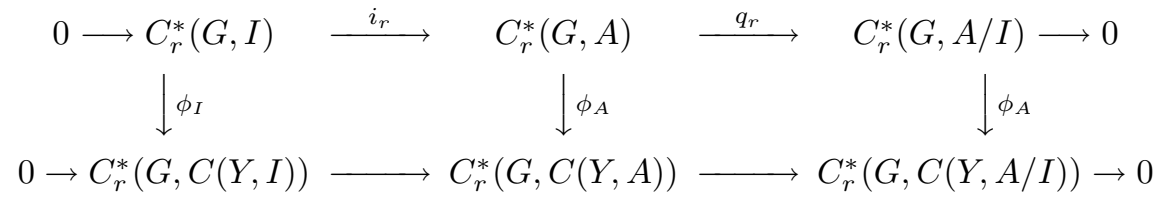


where the bottom line is exact and the vertical arrows are the inclusions induced by the corresponding $G$-equivariant embeddings of $I, A$ and $A / I$ into $C(Y, I), C(Y, A)$ and $C(Y, A / I)$ respectively.

Let us show that the first line is exact in the middle. Let $a \in C_{r}^{*}(G, A)$ be such that $q_{r}(a)=0$. Then we have $\phi_{A}(a) \in C_{r}^{*}(G, C(Y, I))$. Given an approximate unit $\left(u_{\lambda}\right)$ for $I$, the net $\left(\phi_{I}\left(u_{\lambda}\right)\right)$ is an approximate unit of multipliers of $C_{r}^{*}(G, C(Y, I))$. It follows that

$$
\phi_{A}(a)=\lim _{\lambda} \phi_{I}\left(u_{\lambda}\right) \phi_{A}(a)=\lim _{\lambda} \phi_{I}\left(u_{\lambda} a\right) \in \phi_{I}\left(C_{r}^{*}(G, I)\right),
$$

and thus $a \in C_{r}^{*}(G, I)$ since $\phi_{A}$ is injective.

$(2) \Rightarrow(3)$. Let $B$ be an exact $G$ - $C^{*}$-algebra and let $0 \rightarrow I \rightarrow A \rightarrow A / I \rightarrow 0$ be a short exact sequence. Then

$$
0 \rightarrow I \otimes B \rightarrow A \otimes B \rightarrow A / I \otimes B \rightarrow 0
$$

is an exact sequence of $G$ - $C^{*}$-algebras where $G$ acts trivially on the first component of each tensor product. By (2),

$$
0 \rightarrow C_{r}^{*}(G, I \otimes B) \rightarrow C_{r}^{*}(G, A \otimes B) \rightarrow C_{r}^{*}(G, A / I \otimes B) \rightarrow 0
$$

is an exact sequence, and we just have to observe that it coincides with the following one:

$$
0 \rightarrow I \otimes C_{r}^{*}(G, B) \rightarrow A \otimes C_{r}^{*}(G, B) \rightarrow A / I \otimes C_{r}^{*}(G, B) \rightarrow 0 .
$$

$(3) \Rightarrow(4)$ is obvious.

7.3. Theorem. Let $G$ be a locally compact group with property (W) such that $C_{r}^{*}(G)$ is exact. Then $G$ is amenable at infinity.

Proof. We follow the same pattern as in the proof of $(2) \Rightarrow(1)$ given in Theorem 5.8, using here Kirchberg's characterization of exactness (see [Ki] or [Wa]): there exists a net $\left(\Phi_{i}=Q_{i} \circ P_{i}\right)$ of completely positive contractions from $C_{r}^{*}(G)$ into $\mathcal{B}\left(L^{2}(G)\right)$, where $P_{i}: C_{r}^{*}(G) \rightarrow M_{n_{i}}(\mathbb{C})$ and $Q_{i}: M_{n_{i}}(\mathbb{C}) \rightarrow \mathcal{B}\left(L^{2}(G)\right)$ are completely positive contractions, such that for every $a \in C_{r}^{*}(G), \lim _{i}\left\|\Phi_{i}(a)-a\right\|=0$. Moreover, by Corollary 5.7, the maps $\Phi_{i}$ can be chosen with compact support.

Let $\varepsilon>0$ and a compact subset $K$ of $G$ be given. We want to show the existence of a positive type kernel $h \in C_{b, \theta}(G \times G)$, supported in a tube, such that $\mid h(s, t)-$ $1 \mid \leq \varepsilon$ for all $(s, t)$ with $s^{-1} t \in K$.

We first choose a bounded continuous positive type function $f$ on the group $G \times G$, properly supported such that

$$
\forall s \in F, \quad|f(s, s)-1| \leq \varepsilon / 3 .
$$

Let $\Phi: C_{r}^{*}(G) \rightarrow \mathcal{B}(H)$ be a completely positive contraction with compact support, and let $\xi \in L^{2}(G)$. We define $h$ on $G \times G$ by

$$
h(s, t)=\left\langle\rho_{s} \xi, \Phi\left(\tilde{f}_{s^{-1} t}\right) \rho_{t} \xi\right\rangle,
$$

where $\rho$ denotes the right regular representation of $G$ and $\tilde{f}_{s}(t)=(1 / \sqrt{\Delta(t)}) f(s, t)$. Then $h$ is a bounded continuous positive type kernel, supported in a tube $T_{F}=$ $\left\{(s, t) \in G \times G: s^{-1} t \in F\right\}$ where $F$ is a compact subset of $G$ depending on the supports of $\Phi$ and $f$. The main point is to show that $h \in C_{b, \theta}(G \times G)$, in other words, that $g=h \circ \theta$ extends continuouly to $\beta^{u} G \times G$. Recall that

$$
g(s, t)=h\left(s^{-1}, s^{-1} t\right)=\left\langle\rho_{s^{-1}} \xi, \Phi\left(\tilde{f}_{t}\right) \rho_{s^{-1} t} \xi\right\rangle .
$$


Set $M=\sup _{t \in F}\left\|\tilde{f}_{t}\right\|_{r}$. Fixing $t \in G$, let us show first that $s \mapsto g(s, t)$ belongs to $C_{b}^{u}(G)$. This is a consequence of the following computation:

$$
\begin{aligned}
\mid g(u s, t) & -g(s, t)|=|\left\langle\rho_{s^{-1} u^{-1}} \xi, \Phi\left(\tilde{f}_{t}\right) \rho_{s^{-1} u^{-1} t} \xi\right\rangle-\left\langle\rho_{s^{-1}} \xi, \Phi\left(\tilde{f}_{t}\right) \rho_{s^{-1} t} \xi\right\rangle \mid \\
& \leq\left\|\rho_{s^{-1} u^{-1}} \xi-\rho_{s^{-1}} \xi\right\|\left\|\tilde{f}_{t}\right\|_{r}\|\xi\|+\left|\left\langle\rho_{s^{-1}} \xi, \Phi\left(\tilde{f}_{t}\right)\left(\rho_{s^{-1} u^{-1} t} \xi-\rho_{s^{-1} t} \xi\right)\right\rangle\right| \\
& \leq M\left\|\rho_{u^{-1}} \xi-\xi\right\|\|\xi\|+M\|\xi\|\left\|\rho_{u^{-1} t} \xi-\rho_{t} \xi\right\| .
\end{aligned}
$$

Therefore, we may extend $g$ to a function, still denoted by $g$, on $\beta^{u} G \times G$, such that $x \mapsto g(x, t)$ is continuous on $\beta^{u} G$ for every $t \in G$. Let us show now the continuity of $g$ in $\left(x_{0}, t_{0}\right) \in \beta^{u} G \times G$. We write, for $x \in \beta^{u} G, s, t \in G$,

$$
\left|g(x, t)-g\left(x_{0}, t_{0}\right)\right| \leq\left|g(x, t)-g\left(x, t_{0}\right)\right|+\left|g\left(x, t_{0}\right)-g\left(x_{0}, t_{0}\right)\right|,
$$

and

$$
\begin{aligned}
g(s, t) & -g\left(s, t_{0}\right)=\left\langle\rho_{s^{-1}} \xi, \Phi\left(\tilde{f}_{t}\right) \rho_{s^{-1} t} \xi\right\rangle-\left\langle\rho_{s^{-1}} \xi, \Phi\left(\tilde{f}_{t_{0}}\right) \rho_{s^{-1}} \xi\right\rangle \\
& =\left\langle\rho_{s^{-1}} \xi, \Phi\left(\tilde{f}_{t}-\tilde{f}_{t_{0}}\right) \rho_{s^{-1}} \xi\right\rangle+\left\langle\rho_{s^{-1}} \xi, \Phi\left(\tilde{f}_{t_{0}}\right)\left(\rho_{s^{-1} t} \xi-\rho_{s^{-1} t_{0}} \xi\right)\right\rangle .
\end{aligned}
$$

Therefore, we get

$$
\left|g(s, t)-g\left(s, t_{0}\right)\right| \leq\|\xi\|^{2}\left\|\tilde{f}_{t}-\tilde{f}_{t_{0}}\right\|_{r}+M\|\xi\|\left\|\rho_{t} \xi-\rho_{t_{0}} \xi\right\| .
$$

It follows that given $\varepsilon_{1}>0$, there exists a neighbourhood $W_{0}$ of $t_{0}$ in $G$ such that for all $t \in W_{0}$ and all $s \in G$, we have $\left|g(s, t)-g\left(s, t_{0}\right)\right| \leq \varepsilon_{1} / 2$. This inequality remains true for all $x \in \beta^{u} G$, since $g$ is continuous in the first variable and $G$ is dense in $\beta^{u} G$. Finally, we may choose a neighbourhood $U_{0}$ of $x_{0}$ such that $\left|g\left(x, t_{0}\right)-g\left(x_{0}, t_{0}\right)\right| \leq \varepsilon_{1} / 2$ for all $x \in U_{0}$, and therefore we get $\left|g(x, t)-g\left(x_{0}, t_{0}\right)\right| \leq$ $\varepsilon_{1}$ if $(x, t) \in U_{0} \times W_{0}$.

To conclude we note that exactly as in the proof of Theorem 5.8, we can choose $\Phi$ and $\xi$ so that $|h(s, t)-1| \leq \varepsilon$ for all $(s, t)$ with $s^{-1} t \in K$.

7.4. Remark. All almost connected locally compact groups are amenable at infinity but, as already observed, they do not satisfy property (W) unless they are amenable. We do not know if the exactness of $C_{r}^{*}(G)$ is enough to imply the amenability at infinity of $G$.

Note that for a discrete group, all the natural notions of exactness stated in Theorem 7.2 are equivalent. The fact that (4) implies the nuclearity of $C_{r}^{*}\left(\beta^{u} G \rtimes G\right)$ was obtained directly by Ozawa $\mathrm{Oz}$, without using our Theorem 5.8 (see also [GK]). The equivalence between (2) and (4) is a result of Kirchberg and Wassermann [KW].

\section{The CASE OF DisCRETE GROUP ACTIONS}

In this section $G$ will be a discrete group. Our proof in Theorem 5.8 showing that the nuclearity of $C_{r}^{*}(X \rtimes G)$ implies the amenability of the transformation group only involves completely positive maps and positive type functions. On the other hand, in our proof of the converse given in Theorem 5.3, we use representation theory and the characterization of nuclearity in terms of $C^{*}$-norms on tensor products. In certain situations, such as entropy theory, it can be useful to have more explicit proofs, where completely positive finite rank maps approximating the identity are actually constructed.

In [SS], Sinclair and Smith studied the crossed product $C^{*}(G, A)$, for an action of an amenable discrete group $G$ on a $C^{*}$-algebra $A$ having the Haagerup completely bounded approximation property (CBAP). In order to prove the invariance of the 
Haagerup constant $\Lambda(A)$ under crossed products by amenable discrete groups, they constructed explicitly finite rank, completely bounded maps on $C^{*}(G, A)$, approximating the identity map of $C^{*}(G, A)$. In fact, their approach can be easily adapted to get that their result remains true for every strongly amenable $G$-action of any discrete group, and also to prove directly that $(1) \Rightarrow(4)$ in Theorem 5.3.

We keep as much as possible the notation of the paper by Sinclair and Smith. Assume that $A$ is a $G$ - $C_{0}(X)$-algebra with $(X, G)$ amenable, and denote by $\alpha$ the $G$-action. We consider a faithful nondegenerate representation of $A$ in a Hilbert space $H$ such that the $G$-action is implemented by a unitary representation $t \mapsto u_{t}$ of $G$. We set $v_{t}=\rho_{t} \otimes u_{t}$ where $\rho$ is the right regular representation of $G$. Given a bounded function $f: G \mapsto C_{0}(X)$, we define the bounded operator $m_{f}$ on $\ell^{2}(G, H)$ by

$$
m_{f} \xi(s)=\alpha_{s}^{-1}(f(s)) \xi(s), \quad \forall \xi \in \ell^{2}(G, H) .
$$

Assume that $f$ has finite support. For $x \in \mathcal{B}\left(\ell^{2}(G, H)\right)$, we set

$$
T_{f}(x)=\sum_{t \in G} v_{t} m_{f} x m_{f}^{*} v_{t}^{*} .
$$

An elementary computation shows that, if $I$ denotes the identity operator, then $T_{f}(I)=m_{g}$, where $g$ is the constant function on $G$, whose value is the scalar product $\langle f, f\rangle \in C_{0}(X)$ in the Hilbert $C^{*}$-module $\ell^{2}(G) \otimes C_{0}(X)$. In particular, $T_{f}$ is a completely positive map and $\left\|T_{f}\right\|=\|f\|_{2}^{2}:=\sup _{x \in X} \sum_{t \in G}|f(x, t)|^{2}$.

Given a finite subset $F$ of $G$ we denote by $P_{F}$ the projection from $\ell^{2}(G)$ onto the vector space generated by the elements $\delta_{t}, t \in F$, where $\delta_{t}$ is the Dirac mass at $t$.

8.1. Lemma. Let $\Phi: A \rightarrow A$ be a completely bounded map, $f: G \rightarrow C_{0}(X)$ with finite support, and let $F$ be a finite subset of $G$. Then for every $a \in A$ and $s \in G$, the sum

$$
\sum_{t \in G} v_{t} m_{f}(I \otimes \Phi)\left(\left(P_{F} \otimes I\right) \pi(a) \lambda_{s}\left(P_{f} \otimes I\right)\right) m_{f}^{*} v_{t}^{*}
$$

converges in the strong operator topology to

$$
\sum_{t \in F \cap s F} \pi\left(f(t) \alpha_{s}\left(f\left(s^{-1} t\right)^{*}\right) \alpha_{t}\left(\Phi\left(\alpha_{t^{-1}}(a)\right)\right)\right) \lambda_{s} .
$$

Proof. The computation is the same as in the proof of [SS, Lemma 3.2]. Here $\pi$ is the representation defined on $\ell^{2}(G) \otimes H$ by $\pi(a) \xi(t)=\alpha_{t^{-1}}(a) \xi(t)$, and we write $\lambda_{s}$ instead of $\lambda_{s} \otimes 1_{H}$.

In particular, we see that $x \mapsto T_{f}\left((I \otimes \Phi)\left(\left(P_{F} \otimes I\right) x\left(P_{F} \otimes I\right)\right)\right)$ is a completely bounded map from $C_{r}^{*}(G, A)$ into itself; it has finite rank if $\Phi$ has finite rank. It is completely positive if $\Phi$ is.

8.2. Proposition. Let $(X, G)$ be an amenable transformation group and let $A$ be a nuclear $G-C_{0}(X)$-algebra. Then $C_{r}^{*}(G, A)$ is nuclear.

Proof. Given $\varepsilon>0, a_{1}, \ldots, a_{m} \in A$ and $s_{1}, \ldots, s_{n} \in G$, let us construct a finite rank completely positive contraction $\Psi: C_{r}^{*}(G, A) \rightarrow C_{r}^{*}(G, A)$ such that

$$
\left\|\Psi\left(\pi\left(a_{i}\right) \lambda_{s_{j}}\right)-\pi\left(a_{i}\right) \lambda_{s_{j}}\right\| \leq \varepsilon, \quad \text { for } \quad 1 \leq i \leq m, 1 \leq j \leq n .
$$


Using an approximate unit in $C_{0}(X)$ we may assume that there exist a compact subset $K$ of $X$ and a continuous function $\varphi$ with support in $K$ such that $\varphi a_{i}=a_{i}$ for $i=1, \ldots, m$. Let $\eta=\varepsilon /\left(1+\max \left\{\left\|\pi\left(a_{i}\right)\right\|: 1 \leq i \leq m\right\}\right)$. Thanks to Proposition 2.5 , we can choose a function $f: G \rightarrow C_{c}(X)$ with $\|f\|_{2}^{2} \leq 1$ and finite support $C$ such that

$$
\left|\sum_{t \in G} f(x, t) \overline{f\left(s_{j}^{-1} x, s_{j}^{-1} t\right)}-1\right| \leq \eta \quad \text { for } \quad x \in K, j=1, \ldots, n,
$$

where $f(x, t)=f(t)(x)$.

Then we set $F=C \cup s_{1}^{-1} C \cup \cdots \cup s_{n}^{-1} C$ and we choose a finite rank completely positive contraction $\Phi: A \rightarrow A$ such that

$$
\left\|\Phi\left(\alpha_{t^{-1}}\left(a_{i}\right)\right)-\alpha_{t^{-1}}\left(a_{i}\right)\right\| \leq \eta\left(\sum_{t \in G}\|f(t)\|^{2}\right)^{-1}
$$

for $t \in F$ and $1 \leq i \leq m$. It follows that

$$
\left\|\pi\left(\alpha_{t}\left(\Phi\left(\alpha_{t^{-1}}\left(a_{i}\right)\right)\right)\right)-\pi\left(a_{i}\right)\right\| \leq \eta\left(\sum_{t \in G}\|f(t)\|^{2}\right)^{-1}
$$

for $t \in F$ and $1 \leq i \leq m$. For $x \in C_{r}^{*}(G, A)$ we define

$$
\Psi(x)=T_{f}\left((I \otimes \Phi)\left(P_{F} \otimes I\right) x\left(P_{F} \otimes I\right)\right),
$$

and as in the proof of [SS, Theorem 3.4], one shows that

$$
\left\|\Psi\left(\pi\left(a_{i}\right) \lambda_{s_{j}}\right)-\pi\left(a_{i}\right) \lambda_{s_{j}}\right\| \leq \varepsilon \quad \text { for } \quad 1 \leq i \leq m, 1 \leq j \leq n .
$$

Indeed, we write

$$
\begin{aligned}
\| \Psi\left(\pi\left(a_{i}\right) \lambda_{s_{j}}\right)- & \pi\left(a_{i}\right) \lambda_{s_{j}} \| \\
\leq & \left\|\sum_{t \in F \cap s_{j} F} \pi\left(f(t) \alpha_{s_{j}}\left(f\left(s_{j}^{-1} t\right)^{*}\right)\right)\left(\pi\left(\alpha_{t}\left(\Phi\left(\alpha_{t^{-1}}\left(a_{i}\right)\right)\right)\right)-\pi\left(a_{i}\right)\right) \lambda_{s_{j}}\right\| \\
& \quad+\left\|\sum_{t \in F \cap s_{j} F} \pi\left(f(t) \alpha_{s_{j}}\left(f\left(s_{j}^{-1} t\right)^{*}\right)-1\right) \pi\left(a_{i}\right) \lambda_{s_{j}}\right\| \\
\leq & \left(\sum_{t \in F \cap s_{j} F}\|f(t)\|\left\|f\left(s_{j}^{-1} t\right)^{*}\right\|\right) \eta\left(\sum_{t \in G}\|f(t)\|^{2}\right)^{-1}+\eta\left\|\pi\left(a_{i}\right)\right\| \\
\leq & \left(1+\left\|\pi\left(a_{i}\right)\right\|\right) \eta \leq \varepsilon,
\end{aligned}
$$

where we use the Cauchy-Schwarz inequality in the first part of the expression $(*)$.

To conclude, we note that $\Psi$ is obviously a contraction.

Let us recall that for a $C^{*}$-algebra $A, \Lambda(A)$ denotes the infimum of the numbers $\lambda>0$ for which there exists a net of finite-rank completely bounded maps $\Phi_{i}: A \rightarrow$ $A$ converging to the identity in the point norm topology such that $\left\|\Phi_{i}\right\|_{c b} \leq \lambda$ for all $i$. In the same way we get:

8.3. Proposition. Let $(X, G)$ be an amenable transformation group and let $A$ be a $G$ - $C_{0}(X)$-algebra. Then $\Lambda\left(C_{r}^{*}(G, A)\right)=\Lambda(A)$.

8.4. Remark. The same result was obtained in AD2 in the framework of von Neumann algebras for every amenable action of a locally compact group on a von Neumann algebra. For the case of an action of an amenable locally compact group, we also refer to $[\mathrm{HK}$. 


\section{Problems}

9.1. We observed in Section 4 that every inner amenable locally compact group has property (W). We do not know if property (W) can be strictly weaker. For almost connected groups or Type I groups, property (W) (as well as inner amenability) is equivalent to amenability (see Remark 5.10). On the other hand, every discrete group has property (W). Therefore, if there exist examples of locally compact groups with property $(\mathrm{W})$ that are not inner amenable, they should be investigated in the class of nondiscrete non-type-I totally disconnected groups.

9.2. As mentioned in Section 6, the right definition of an amenable $G$ - $C^{*}$-algebra is still unclear, and many problems remain unsolved. The notion of strong amenability introduced in Definition 6.1 is quite natural, but might be too strong for some purposes. It is tempting to define a $G$ - $C^{*}$-algebra $A$ to be amenable if and only if $C^{*}(G, A)=C_{r}^{*}(G, A)$. One would like to solve the following questions:

(a) If $A$ is a nuclear $G$ - $C^{*}$-algebra, does the equality $C^{*}(G, A)=C_{r}^{*}(G, A)$ imply that $C_{r}^{*}(G, A)$ is nuclear?

(b) Does the equality $C^{*}(G, A)=C_{r}^{*}(G, A)$ remain true for the restriction to any closed subgroup?

(c) Is the property $C^{*}(G, A)=C_{r}^{*}(G, A)$ functorial in an appropriate sense? This would solve question (a). The strongest reasonable functoriality property is the following one: given any $G$ - $C^{*}$-algebra $B$ and any $G$-equivariant morphism $\Phi: A \rightarrow M(B)$ such that $\overline{\Phi(A) B}=B$ and $\Phi(Z(M(A))) \subset Z(M(B))$, does the equality $C^{*}(G, A)=C_{r}^{*}(G, A)$ imply that $C^{*}(G, B)=C_{r}^{*}(G, B)$ ?

(d) If $A$ is a simple $G$ - $C^{*}$-algebra, does the equality $C^{*}(G, A)=C_{r}^{*}(G, A)$ imply that $G$ is amenable?

Even in the case of a discrete group $G$ acting on a commutative $C^{*}$-algebra $C_{0}(X)$, we do not know if $C^{*}(X \rtimes G)=C_{r}^{*}(X \rtimes G)$ implies the nuclearity of $C_{r}^{*}(X \rtimes G)$. However, we know that $C_{r}^{*}(X \rtimes G)$ is nuclear if and only if $(X, G)$ is an amenable $G$-space, and therefore if and only if the $G$ - $C^{*}$-algebra $C_{0}(X)$ is strongly amenable (see Theorem 5.3 and Proposition 6.3).

Some conditions sufficient to ensure that $C^{*}(G, A)=C_{r}^{*}(G, A)$ for a not necessarily commutative $C^{*}$-algebra $A$ have been studied in [Ex], [EN].

Let us note that already in the case of proper actions, there is no obvious definition of a proper $G$ - $C^{*}$-algebra $A$ when $A$ is not commutative. For a comprehensive analysis of this question we refer to [Ri].

9.3. For any locally compact group, does the exactness of $G$ imply that $G$ acts amenably on a compact space?

Is the exactness of $G$ equivalent to the exactness of its reduced $C^{*}$-algebra? (See the concluding remarks in $[\mathrm{KW}$.)

Acknowledgement. I would like to thank J. Renault for valuable discussions concerning property $(\mathrm{W})$ and amenability at infinity.

\section{REFERENCES}

[Ad] S. Adams: Boundary amenability for word hyperbolic groups and an application to smooth dynamics of simple groups, Topology, 33 (1994), 765-783. MR 96g:58104

[AD1] C. Anantharaman-Delaroche: Systèmes dynamiques non commutatifs et moyennabilité, Math. Ann., 279 (1987), 297-315. MR 89f:46127 
[AD2] C. Anantharaman-Delaroche: Amenable correspondences and approximation properties for von Neumann algebras, Pacific J. Math., 171 (1995), 309-341. MR 96m:46106

[ADR] C. Anantharaman-Delaroche and J. Renault: Amenable groupoids, Monographie de L'Enseignement Mathématique 36, Genève, 2000. MR 2001m:22005

[Co] A. Connes: Classification of injective factors, Ann. of Math., 104 (1976), 73-115. MR 56:12908

[Di] J. Dixmier: Les $C^{*}$-algèbres et leurs représentations, Gauthier-Villars, Paris, 1964. MR 30:1404

[Ef] E. G. Effros: Property $\Gamma$ and inner amenability, Proc. Amer. Math. Soc., 47 (1975), 483486. MR 50:8100

[Ex] R. Exel: Amenability for Fell bundles, J. Reine Angew. Math., 492 (1997), 41-73. MR 99a:46131

[EN] R. Exel, C. K. Ng: Approximation property of $C^{*}$-algebraic bundles, Preprint 1999.

[Fu] H. Furstenberg: Boundary theory and stochastic processes in homogeneous spaces, in Harmonic analysis on homogeneous spaces, Proc. Sympos. Pure and Appl. Math. 26 (1973), 193-229. MR 50:4815

[Gre] F. P. Greenleaf: Invariants means on topological groups, Van Nostrand, New York, 1969. MR 40:4776

[Gro] M. Gromov: Spaces and questions, Geom. Funct. Anal. 2000, 118-161. MR 2002e:53056

[GK] E. Guentner and J. Kaminker: Exactness and the Novikov conjecture, Topology, 41 (2002), $411-418$.

[Gui] A. Guichardet: Tensor products of $C^{*}$-algebras, Aarhus University Lecture Notes Series No. 12, 1969.

[HK] U. Haagerup and J. Kraus: Approximation properties for group $C^{*}$-algebras and group von Neumann algebras, Trans. Amer. Math. Soc., 344 (1994), 667-699. MR 94k:22008

[Hi] N. Higson: Bivariant $K$-theory and the Novikov conjecture, Geom. Funct. Anal., 10 (2000), 563-581. MR 2001k:19009

[HR] N. Higson and J. Roe: Amenable group actions and the Novikov conjecture, J. Reine Angew. Math., 519 (2000), 143-153. MR 2001h:57043

[Ki] E. Kirchberg: On non-semisplit extensions, tensor products and exactness of group $C^{*}$ algebras, Invent. Math., 112 (1993), 449-489. MR 94d:46058

[KW] E. Kirchberg and S. Wassermann: Exact groups and continuous bundles of $C^{*}$-algebras, Math. Ann., 315 (1999), 169-203. MR 2000i:46050

[La1] E. C. Lance: On nuclear $C^{*}$-algebras, J. Functional Analysis, 12 (1973), 157-176. MR 49:9640

[La2] E. C. Lance: Hilbert $C^{*}$-modules. A toolkit for operator algebraists, London Math. Soc. Lecture Note Series 210, Cambridge Univ. Press, 1995. MR 96k:46100

[LP] A. T. M. Lau and A. L. T. Paterson: Inner amenable locally compact groups, Trans. Amer. Math. Soc., 325 (1991), 155-169. MR 91h:43002

[LR] V. Losert and H. Rindler: Conjugate invariant means, Colloq. Math., 15 (1987), 221-225. MR 89d:43001

[MZ] D. Montgomery and L. Zippin: Topological transformation groups, Interscience Publishers, 1955. MR 17:383b

[Oz] N. Ozawa: Amenable actions and exactness for discrete groups, C. R. Acad. Sci. Paris Sér. I Math., 330 (2000), 691-695. MR 2001g:22007

[Pa1] A. L. T. Paterson: Amenability, Mathematical Surveys and Monographs No. 29, Amer. Math. Soc., Providence, RI, 1988. MR 90e:43001

[Pa2] A. L. T. Paterson: The class of locally compact groups $G$ for which $C^{*}(G)$ is amenable, in Harmonic analysis (Luxembourg, 1987), Lectures Notes in Math., 1359 (1988), Springer, 226-237. MR 90m:22019

[Pe] G. K. Pedersen: $C^{*}$-algebras and their automorphism groups, Academic Press, 1979. MR 81e: 46037

[Re] J. Renault: A groupoid approach to $C^{*}$-algebras, Lecture Notes in Math., 793 Springer, Berlin, 1980. MR 82h:46075

[Ri] M. A. Rieffel: Integrable and proper actions on $C^{*}$-algebras, and square-integrable representations of groups, Preprint 1999.

[SS] A. M. Sinclair and R. R. Smith: The completely bounded approximation property for discrete crossed products, Indiana Univ. Math. J., 46 (1997), 1311-1322. MR 99e:46072] 
[STY] G. Skandalis, J.-L. Tu and G. Yu: Coarse Baum-Connes conjecture and groupoids, Preprint 1999.

[Wa] S. Wassermann: Exact $C^{*}$-algebras and related topics, Lecture Notes Series 19, Global Analysis Research Center, Seoul National University, 1994. MR 95b:46081

[Yu] G. Yu: The coarse Baum-Connes conjecture for spaces which admit a uniform embedding into Hilbert space, Invent. Math., 139 (2000), 201-240. MR 2000j:19005

Département de Mathématiques, Université d'Orléans, B. P. 6759, F-45067 Orléans Cedex 2, France

E-mail address: claire@labomath.univ-orleans.fr 